$Q \mathrm{~L}$

430.4

T18

1808

MOLL.

$$
\begin{aligned}
& 594.08 \\
& . T 184
\end{aligned}
$$




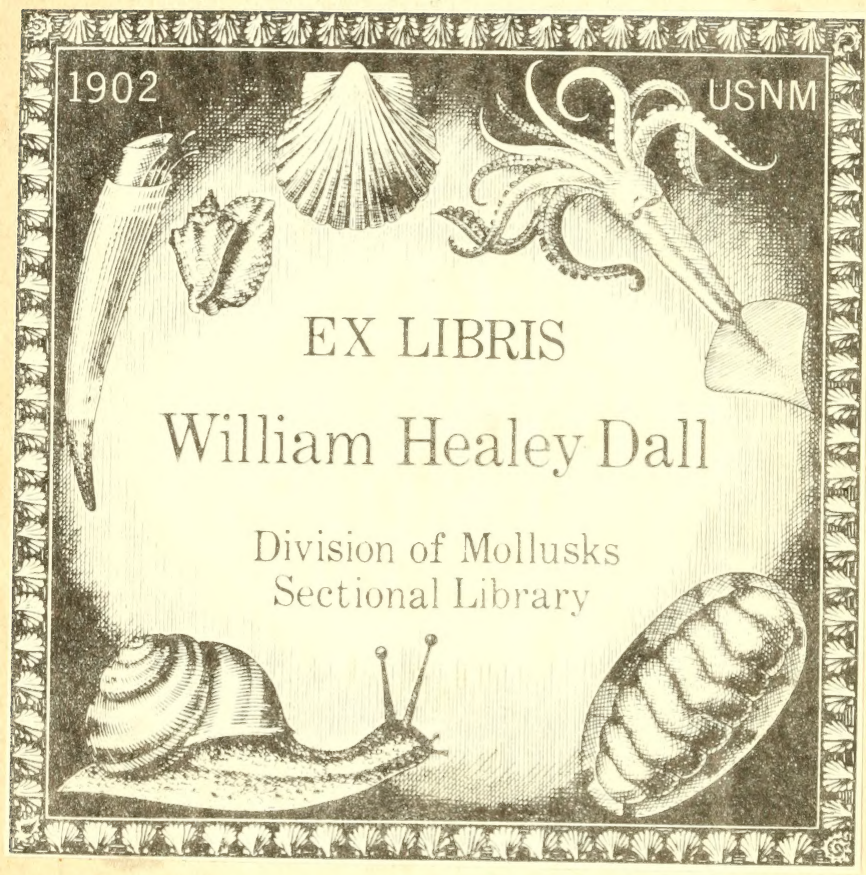




\section{Division of Mollusks} Sectional Library 

S- TAKEEN

LA COCHLIOPERIE. 
Se trouve a $P A R$ Ls

Caez Petit, Libraine, Palais du Tribunat, $n^{\circ} \cdot 257$.

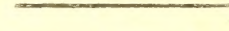

A V $\mathrm{IS}$.

Tout Exemplaire de cet ouvrage, qui ne sera pas revitu de la signature de 7'Auteur ì la page 5, et de celle de l'17nprimeur aubas de cet Avis, sera une contrefacon.

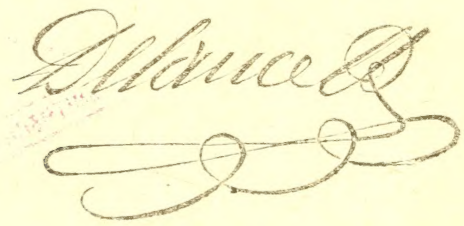




\section{LA COCHLIOPERIE, RE C UEIL}

D'EXPÉRIENCES TRÈS-CURIEUSES SUR LES

\section{HËLICES TERRESTRES,}

VULGAIREMENT NOMMÉS ESCARGOTS;

AVEC UNE INSTRUCTION

SUR LA GUÉRISON RADICALE

\section{DES HERNIES OU DESCENTES,}

Sans dépense, ni aucun secours étranger.

PAR GEORGE TARENNE.

$$
\begin{aligned}
& \text { Prix, 6fr. } \\
& \qquad \begin{array}{l}
34805- \\
\text { Jि14 } 81890
\end{array} \\
& \text { PARIS, }
\end{aligned}
$$

DE LIIMPRIMERIE DE DELANCE.

1808. 



$$
\begin{gathered}
\text { a } \\
\text { La Shilosophies } \\
\text { Et à } \\
\text { L'Sumanité. }
\end{gathered}
$$

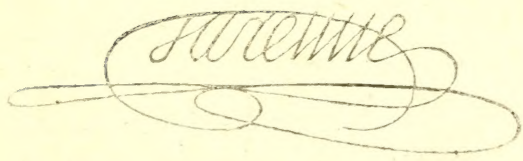





\section{LA COCHLIOPERIE。}

PREMIERE PARTIE,

SUR LES REPRODUCTIONS ANIMALES.

\section{ARTICLE PREMIER。}

DE LA SCIENCE ET DE L'ART.

$\mathrm{O}_{\mathrm{N}}$ lit, dans quelques auteurs, dont je citerai les plus célèbres, que le colimaçon de terre, ou escargot, a la faculté de vivre sans tête; et cqu'il la reproduit, quelque tems après cqu'on la lui a coupée.

Le naturaliste Spallanzani, de Modèxe, passe pour être le premier qui ait fait cette observalion. Il affirme, dans 


\section{IA COCHLIOPERTE.}

son Programme ou Précis d'un ouvrage sur les reproductions animales, imprime en italien et traduit en francais, vers le: commencement et la fin de la mème année I768, cque la têle d'un escargok se reproduit, comme les ongles et les rrilfes, dans les animaux bipedes et quadrupedes. Ayant, dit-il, coupé la tîte à plusieurs escargots, toutes les parties de celte tête se sont reproduites, ei ont forme, a bout d'un cerlain tems, un fout assez resscmblant à leur premier modèle, ou à la tête préexistante.

Valmont de Bomare assure, au coniraire, dans son Dictionnaire d'Hisioire naturelie, au nut Limacon, cqu'ayant tenié, sur le témuignage de Spallanzani. et le bruit public, nombre d'expériences ì ce sujet, durant l'automne de r760, lous les escargots dont il avait sciparé la lête d'un seul coup de conteau, taicnt morts au bout de quelques jours, exceptés 


\section{LA COCHLIOPERIE.}

deux, qui, a près être restés cinq mois col. lés contre une muraille, pleins de vie, moururent enfin au printems, sans aucune. apparence de reproduction de tète. Ceux, dit-il, auxquels je ne coupai pas la tête avec assez de précipitation, sortirent effectivement de leur coquille, au bout de dix ou douze jours, portant des cornes mutilées; mais ceux auxquels je coupai brusquement la moitié diagonale de la tête, quoiqu'ils ressortissent aussi de leur coquille, au bout de quelques jours, ils ne rampèrent jamais qu'avec deux seules cornes. Valmont de Bomare ajoute qu'il a répété ces expériences en 1769 , et qu'elles ont toujours été sans aucun succès. Quantité de persomnes lui ayant écrit, de dirers pays, pour lui faire part que leurs tentatires avaient été semblables aux sienues, il en a conclu que, si l'expérience a réussi à Spallanzani, c'est qu'il h'a pas mis une assez grande célérité 


\section{to LA COCHLTOPERTE}

dans l'amputation, ce cu'il n'a conpú cque la peau de l'animal.

Voltaire, que je vais copier littéralement, dit, dans ses Questions sur l'Enw cyclopédie, au mot Colimacons: «Le 27 " de mai, 1768 , je coupai la téle entiere, "arec ses quatre antennes, it vingt liw "maces nues incoques, de couleur mor. " doré brun, et à douze escargots à co"quilles. Je coupai aussi la tele à huit "s autres escargols, mais entre les anten"nes." (C'est-ì-dire, qu'il lemr coupa les "s deux petites cormes et lez lexres.) "Au "bout de quinze jours, deur de nues lis maces montrèrent une lite naissante, " elles mangeaient déjà, cf leurs gruatre " antennes commençaient à poindre. "Les autres se portent bien; dies.man"gent sous le capuchon qui les convre, " sans allongrer le cou. Il n'est nion! que " la moilić des escargots, tous lis autres $"$ sont en vie. Ils marchent, ils grim- 
:pent à un mur, ils allongent le cou; " mais il u’y a mulle apparence de tête, " excepié à un seul. Cependant je doute " si cet escargot, auquel il revient une " iéte, et clont une corne commence à " paraitre, u’est pas du nombre de ceux " ̀̀ quni je fis l'amputation entre les an" iennes. Tons ceux-ci onl dejà repris " livur museau. Dès cqu'ils seront en état " de manger et de faire l'amour, j'en "rendrai compte. J'ose espérer que mes " escargots, nies colimaçons, reprenm " diront dies têles entières, comme les "limaces; mis enfin je n'en ai encore " ra qu'un à quir cela soit arrivé, et je " crains bien. de m’être trompé. "

Il est très-probable que les escargots mutilés par Voliaire sont tous morts, puisqu’il ne parle plus du résultat de cette première expérience, dans ses cuvres; et que l'édition d'où je tire ce pas-sagte est de 1775 , c'est-it-dire, postidieure 


\section{I2 IA COCHLTOPERIE.}

de sept années à ses decapitalions. Il affirme seulement, en quelques endroits de cette même edition, que les limaces et les escargols recouvrent une nouvelle tête, au bout d'un mois, plus ou moins; mais il prérient, au mol Serpent de ses Questions Lincyclopédiques, que s'il a fait heureusement deur fois cette expérience, il l'a manquée trente. C'est que probablement, dans les deux fois dont il parle, il n’aroil encore coupé que la peau de la tête. Au reste, Voltaire a-t-il fait nsage, en I 768 , l'un conteau, ou d'une paire de ciseaux? il n'atrait pas dì nous lelaisser ignorer. Pour bien juger du succès de pareilles décollations, il est nécessaire de comnaitre l'instrument dont on s'est servi, et si l'un en a fait usage arec une tres-grande promptitude; car, au moindre atfouchement, l'animal se retire sur lui-même, avec une force et ıne vitesse extraordinaires. 


\section{LA COCHLIOPERTE. IS}

Voltaire, aussi peu satisfait qüil devait l'être de ses premières opérations, les a renouvelées, quatre années après. Il n'y a pas de doute qu'il n'ait fait usage alors d'une paire de ciseaux. Il dit, toujours dans le même ourrage, à l'article Rétractation, qui en termine le dernier rolume : «Ma première rétractation est. "sur les ciseaux avec lesquels j'avais " coupé plusieurs tètes de colimaçons. "Toutes leurs têtes revinrent en I 772;" " (c'est-d̀-dire, dans les expériences qu’il " fit ell $I 772$, avec des ciseaux; ) "mais " celles que je coupai en 1773 , ne sont " jamais revenues." (Ainsi, comme je "l'ai remarqué plus haut, il abancionne " absolument ses expériences de I768.) " "Des gens plus habiles que moi, m'ont "fait aperceroir que, lorsque mes têtes "étaient ressuscitées, je n'arais coupé " que la peau de leur visage, "( parce " qu'il ne faisait pas l'amputation avec 
If LA COCHLIOPERIL

" assez de promptitude,) " et que je n'a" vais pas entamé leur cervelle, qui est " la source de leur vie, tout comme chez " nous." ( Assertion fausse; je le dé" montrerai. ) " Lorsque j’ai coupé la " têle entière, avec plus d'adresse, cette " tête ne s'est point reproduite; mais " c'est toujours beaucoup d'avoir fait " renaître des visages.

Voltaire dit enfin, dans ses Mélanges de Littérature, même édition, article Des Limaçons : « Les expériences sur "les limaces sont les plus étonmantes "qu'on ait faites, et l'on n'est pas au "bout. "Ne voulant pas lui-même s'en tenir là, et ne croyant pas avoir été assez plaisant, lorsqu'il a parlé de résurrections de têtes et de visages d'escargots, il a enrichi la littérature d'un ouvrage singulier, sur ce sujet. Cet ouvrage a pour titre: $L_{E S}$ Colmaçons durévérend père l'Escarbotier, par la grace de Dieu, 


\section{1. $A$ COCHLIOPERIE.}

sapucin indigne, prédicateur ordinaire, et cuisinier du grand couvent de la ville de Clermont, en Auvergne. Mais je laisse la plaisanterie du littérateur, pour rerenir à la prédiction du physicien.

Cette prédiction de Voltaire m'a fait naitre l'idée de renoupeler les expériences dont je viens de parler, et d'en vérifier moi - même les résultats. Je n'ai pu me résoudre à toucher et garder des limaces, qui sont des animaux extrêmement sales. Je me suis borné à opérer sur des escargots, aussi nommés limaçons communs et terrestres, ou mieux, colimaçons, pour coc-limaçous, c'est-àdire, limaçons à coquille. J'ai choisi de préférence l'escargot de vigne, (helix. pornatia,) qui est la plus belle espèce de ce genre de coquillage. J'en ai décapité, égorgé, mutilé un nombre considérable, de toutes les manières, durant dix - hui inois, répétant et diversifiant mes exper- 


\section{I A COCHLIOPERIL.}

riences de quinze en quinze jours. Je compte que j'ai détruit environ un millier de ces paurres bêtes. T’en ai immolé ce nombre prodigieux, parce que j’ai présu qu'il en mourrait heaucoup à la suite de chaque expérience, et parce qu'il ine fallait chercher, dans les survivans, l'époque de l'amée la plus favorable à leur ségénération. Ainsi, mes opérations ayant commencé le premier jour d'un automne, elles ont cluré deux automnes, deux hivers, un printems, et un été. La première demi-amuée a été doublée, parce que mes expériences n’araient pas été faites avec assez de précaution, in sur un assez grand nombre d'escargots, lorsque je commençai calte espèce d'étude amatomique, physiologicque, philosophique et métaphysique.

Je supplie mes lecteurs de ne pas s'imasiner que j’aie fait cet alfrens carnage, sans éprouver un scintinent pénible; la 
compassion a ému mon cœur. Mais j’ai cru que ces sacrifices seraient agréables à la raison, et plus utiles encore à l'humanité. On jugera, par cet ouvrage, si je me suis fait illusion. Quel que soit le sentiment qu'en auront les âmes sensibles, je représenterai qu'il y a beaucoup moins de mal à immoler, en gémissant, des animaux, sur l'autel de la science; que de les tuer, avec plaisir, sur le bloc de l'intempérance, de la gourmandise et de la volupté.

Il s'en faut beaucoup cependant, que japprouve toutes les opérations cruelles qui ont été faites sur les brutes, pour l'instruction de l'homme, particulièrement sur les animaux à voix plaintives. Il est avantageux qu'elles aient étéfaites; je ne voudrais pas en être l'auteur : je voudrais encore moins être du nombre de ceux qui, transportés d'un zèle dénaturé, on: cllerché à acquérir des lu- 
mières, ou à perfectionner leurs arts, en plongeant le glaive dans le corps même de leurs semblables. De ce nombre, on peut mettre Hérophile, médecin grec, natif de Calcédoine en Bithynie, lecquel rivait 300 ans avant J.-C. Il dissécqua, dans l'école d'Alexandrie, les corps vivans de plus de sept cents hommes et femmes, réputés criminels, pour mieux y faire roir les secrets et les merveilles del'anatomie. Personne n’ignore ce qu'on dit de Le Giotio, peintre et archilecte italien, vers le commencement du quatorziène siècle. Voulant représenter un Christ, il engagea un pauvre homme à se laisser attacher à une croix, pondant une heure seulement; il finit par le poiguarder, pour acherer de le peindre en cei état. Parrhasius, fameux peintre d'Ephèse, qui virait près de 400 ans avani J.-C., fut encore plus barbare. Désimant peindre un Prométbée dechiré 


\section{IA COCHLIOPÉRIE,}

par un vautour, il acheta un vieillard corinthien, auquel il fit subir cet horrible supplice, après lui avoir un peu ourert le bas - rentre. Rondelet, médecin de Montpellier, au commencement du seizième siècle, doit être aussi rangé parmi ces monstres, quoique le trait qu'on cite de lui n'ait rapport qu'à un cadarre: il disséqua lui-même un de ses propres enfans.

Voilà de ces actions épouvantables qui arrachent des larmes, qui glacent le sang, qui font dresser les cheveux à la tête. Mais couper le cou à cinquante escargols en un jour, ou manger quatre douzaines d'huitres à son dîner, en vérité, si cela ne revient pas au même, c'est que la déglutition est plus douloureuse que la décapitation.

Sans domner un journal circonstancié de loutes mes expériences, comme l'a fait Haller, dans ses Rtemoires sur la 
nature sensible et irritable des parties animales, je tâcherai de n'omettre aucune des observations qui en ont été les résultats, et de suirre un ordre métho. dique qui, sans être ennuyeux, satisfera plus l'esprit qu'un tableau monotone, chargé de répétitions. Je rapporterą très-exactement tous les procédés dont ¡'ai fait usage, afin qu'on puisse être assuré de la vérité de mes assertions, et les vérifier même, si on le juge à propos. En général, je me suis servi, dans les amputations considérables, de grands ciseaux fins dont le taillant était très-vif, et avait deux pouces et demi de longueur. Dans les autres opérations, je me suis servi de petits ciseaux, ou ciseaux ordinaires, bien tranchans. J'aurai soin de les distinguer dans le cours de nies expériences, lorsque je croirai convenable de ne laisber aucune indécision sur leus usage. 


\section{LA COCHITOPĹRE. 2 I}

\section{A R T I C L E II.}

\section{$T \hat{E} T E$.}

Povr couper habilement la tête à un escargot, on le tient de la main gauche par la coquille, on pose un des taillans d'une paire de grands ciseaux sous sa gorge, et on lui laisse le tems de revenir de ce premier attouchement. Lorsqu'il allonge bien le cou, lorscue ses cornes paraissent pleines de la liqueur noirâtre qui semble être le principe le plus actif de son sentiment, on rapproche peu at peu les ciseaux, jusqu'à ce que la branche supericure soit presque sur le cou du reptile, derricre les grande's cornes. Alors, serrant virement les doig!s, on lui abai la tête entière, arec autant de céiérité qu’on en pourrait mettre à prow noncer la lettre A. 


\section{LA COCHLIOPERIE.}

La tête d'un escargot ainsi coupée, doit demeurer sur les cisenux, ou tomber dans un récipient, avec sez quatre cornes noires, et deux masses intérieures. La plus considérable est la masse de li bouche; elle remplit, pour ainsi dire. toute la tête. L'autre, de forme oblongue, située transversalement sous les grandes cornes, est la cervelle. Ce viscère est grisâtre, un peu aplati sur la masse de la bouche, et ressemblant assez. à une goutte de glaire d'ouf, dans laquelle on aurait mis quelques grains de cendre. Il forme la partie supérieure d'un anneau membraneux presque invisible, au travers ducinel passent la bouche, la langue, la gorge et l'asophage, ou le conduit des alimens. Quelquefois cet anneau se tronve devant la gorge.

Sila tête d'un escargot reste collée aux ciseaux, ou si elle tombe dans un rase, sur sa partie inférieure, c'esi-à-dire, sur 


\section{A COCHLIOPÉRIE. 25}

la bouche et la gorge, elle conserve ses cornes droites, l'espace d'environ cinq minutes, en automne ou en hiver; et pendant près d'une heure, au printems ou en été. Ensuite les cornes se replient peu à peu sur elles-mêmes, et se couchent sur le devant de la tête, de méme que se courbent ordinairement vers la terre, les fleurs qui se famnent et qui meurent. Une tête d'escargot jouit du mouvement et du sentiment, jusqu'à ce que ses cornes soient ainsi abattues : elle les agite doucement, sans y être excitée par aucune cause étrangère ; elle les retire, quand on les touche avec une épingle ou un autre corps quelconque; et dans la belle saison, l'animal est si vigoureux, que la tête repousse naturellement ses cornes, après les avoir retirées.

Ce phénomène de l'économie animale doit être comparé à ceux que l'on remar que dans tous les animaux à sang froid; 


\section{2f IA COCHLIOPLRIE.}

par exemple, à celui qu'offre le cœur des grenouilles et des crapauds. Selort Harrey, que l'on dit être le premier médecin qui ait décourert la circulaíon du sang, le cocur d'une grenouille arraché, puis exposé immédiatement après au soleil, ou mis sur une assietle un peu chaude, s'agite ordinairement pendant une heure. Lorsque son mourement de diastole et de sistole semble être perdu, il suffit de l'irriter avec ume épingle pour le faire battre de nouveau (I).

(1) La Mettrie dit, dans son Art de faire des garcons: "L'Auleur de V'énus physique 2) a attribué un peu légèrement, à son ami "Harvey, la découverte de la circulation du 》sang. Son véritable auleur est, dit-on, Ser" ret, médecin espagnol, que Calsin, malggré 》) cette heureuse invention, fit brûler à Ge"nève, pour affaire de religion. Fra-Paolo 


\section{LA COCHLIOPERIE. 25}

J'aii tenté cette espérience; et après en avoir reconnu la vérité, j’en ai fait plusieurs autres semblables, qque je vais rapporter le plus succinctement cqu'il me sera possible.

Vers le milieu du printems, jai pris, avec des tenailles, par les deur pattes

" ayant appris, par les ourres de cet infor-

"tuné, la nouvelle de la circulation du sang, " en fit part à quelques anglais qui ćtudiaient " à Padoue. Ceux-ci, de retour en leur pays. " la portèrent à Harrey, qui sux-le-champ se " mit à en faire la vérificalion. Peu de lems ) apiès, il publia ses expériences sir ce sujet, ") lesquelles appuyées du crćdil de ses com") patriotes, et de la parlialité de leurs aduv lateurs, lui frent, aux yeux des gens mal ) instruits, usurper, sur ce malheureux Ser"yet, l'honneur de sa découverle."

Voyez aussi le Traité de l'Opinion, par Lo Gendre de Saint-Aubin, lone THI, De L. Mí. DECIXE, arlicle De la Circulation dusang. 


\section{LA COCHLIOPERIE.}

de derrière, une très-grosse grenouille: je l'ai couchée sur le dos : je lui ai fait, sur la peau du rentre, une grande incision cruciale, avec de petits ciseaux : jai dégagé celte peau, qui est très-délicate; et j’ai ourert la poitrine, avec les mêmes ciseaux, sans aflecter les viscères qu'elle renferme. L'animal étant ainsi éventré, jai clégragé les poumons et le çur, que jai laissés ensemble, pour ne pas blesser l'organe qui devait fixer mon attention. Je les ai mis au soleil, sur un petit marbre un peu chauffé. Le mouvement du coeur a été sensible l'espace d'une heure et demie; après quoi je l'ai fait continuer, à plusieurs reprises, pendant une autre demi-heure, en l'écliauffant arec mon halcine. Quant à la grenouille, elle tait trìs-rivace. L'ayant vidée entièrement, ot l'ayant mise dans de l'eat fraîche, elle y a vécu l'espace d'une heure, sautant quelquefois jusqu'i $l_{\text {it }}$ 


\section{LA COCHLTOPERTE.}

hauteur de six ou huit pouees, dans le: vase qui la contenait. Au bout de co tems, je l'ai retirée de l'eau, parce qu'elle y demeurait sans mourement; et $\mathrm{ja}$ a remarqué, arec beaucoup de surprisc, qu'elle fermait encore les yeus, quand je les touchais arec une épingle. Le lendemain, j’ai fait suhir la même opération à une autre grenouille; elle a vécu une heure et demie hors de l'eau. Son cceur, mis à l'ombre et au frais, a conservé son mourement péristaltique pendant plus de cquatre heures, sans l'inciter par aucun atiouchement, ni par aucun moyen quelconque. Une autre fois, j'ai coupé la tête à une troisième grenouille, arec de grands ciseaux; j’ai jeté son corps dans de l'eau, et il y a vécu une heure et demie. J'ai décapité une quatrième grenouille, je l'ai mise dans un pot vide et au frais; elle y a vécu vingt-quatre heures, quoiqu'elle eut perdu ton! son sangु, 


\section{LA COCHLIOPLRIE.}

fuviron cinc minuies après la décoilation.

Ces qua!re rpériences mont ensuite porlé à me fare ha rustion suifante: Ou est le sicuge de la rie dans me grenonille, w proind bloment dans tois les animaux, homnes ou brutes?

Descaries prétend que les létes ne dioireni point itre comparées it l'iomme, parce quielles ne sont que des automates.... Péreira, medecin erparnol. avait enseijné, cont ais avant lui, ce principe absurde ef firuce.... Mais Newton, cui arait pour le moins autant de ruison, de bon sens et de génie, cque ces denx nétaphysicions, diit que le plan de la Nature est unique dans touies ses curres, et quelle esi milorme dans ses moyens: Natura est sibi semper consona; "la Nature est toujours semblable à elle"même. "Salomon dit aussi, clans les Proverbes: "Ie juste connait l'âme des 


\section{A COCHLIOPERIE.}

" bètes, mais les entrailles du méchant "sont cruelles $(*)$. "... Mon esprit et mon cour mayant toujours fait partager ce dernier sentiment, aussi bien quie celui de Nervton; et la méditation m'en ayant fait voir la solidité, la sagesse: ¡ai cru convenable d'examiner scrupuleusement où est le siége de l'âme, et je le demande ici, sans distinction de genre ni d'espèce, dans tout le règue animal ?

Si l'on dit, arec Hippocrate, Chrysippe, Diogene, Aristote, Lucrece, A rerroès, autsement nommé Ebriet plusieurs autres, que l'âme eâ dans le milieu de la poitrine, c'est-i-dirs, dans le cour ; la première et l.x :ecudic expérience prouvent le contraire.

Si l'on dit, arec Epicare cl se: actom teurs, que l'âne est clans l'estonac; la

(*) Proverbes de Salomon, chapitre XIT, verset 10. 
prenière et la seconde expérience piotivent encore le contraire.

Si l'ou dit, avec Pythagore, Erasistrate. Hérophile, Straton, Cicéron, Galien, Cassiodore, Charron, Descartes, Vieussens, Lancisi, la Peyronie, et presque tous les métaphysiciens, que l'âme est dans le cerveau; la troisième et la quatrième expérience prouvent le contraire: les décapitations d'escargots le prouveront encore mieux.

Si l'on dit, avec Noé, Moïse, Empébocle, Critias, Virgile, Lucain, etc., gue l'âme est dans le sang; la première, in seconde, la troisieme et la quatricme expérience s'y opposent également.

Enfin, si l'on prétend, avec le catéchisme des Russes, que l'âme est répandue dans la tête et le coeur; ou bien, arec Platon, que l'âme est divisée en trois paries, saroir : la première, qui est la raison, daus le cerveau; la seconcie. 


\section{LA COCHLIOPERTE. 3.}

qui est la colère, dans la poitrine; et la troisième, qui produit les inclinations roluptueuses, dans les entrailles; il sera toujours facile de prouver que ce sentiment est une erreur.

Eventrez une grenouille, depuis la gorge jusqu'à l'anus; videz entièrement la poitrine et le rentre; coupez-lui la têle; mettez-la clans un pot ou un vase quelconque, qui soit un peu profond, et sans eau : elle y vivra, elle y saulera, pendant une heure et demie, plus ou moins. Cette dernière expérience est très-curieuse, mais elle est effrayante.

Beaucon! de philusophes ont essayé d'expliquer ees cirrers eliets du mécanisme animal. Lucrece, entre autres, en a disserté lonzuemeni, dans son poëme De la Nalure des choses (*). Tont ce

$\left(^{\star}\right)$ De la Nalure des choses, livre III, depruis le vers 638 jusqu'au vess 673. 


\section{LA COCHLTOPÉRIIE。}

qu'ils ont dit ne prouve et n'apprend rien. Le raisonnement de chacun d'eux n'est que le chaos au milieu de l'abîme; et il est très-probable qu'il ne sera jamais donné à l'homme d'en faire sortir la lumière.

Oiu suis-je? qui suis-je? et que deviendrai-je?

Tel sera toujours, jusquà la fin des siècles, le cri du vrai philosophe. La révélation pourra répondreà la troisième question; la métaphysique, tâcher d'analyser la seconde; qutant à la première, il n'ya rien pour notis enseigner la vérité, rien, et tout nous trompe.

S'en suivra-t-il que le mot âme, aux yeux de la seule raison, doit être le nom d'une chimère? Gardez-vous d'une telle pensée! elle serait extravagante : elle le serait sous tant de rapports, qute je n’appréhende pas d'écrire, que celui qui nie ouyertement l'existence st l'im- 


\section{A COCHLTOPERTE. 33}

mortalité de l'âme, fîl-il d'ailleurs un homme de bien, merric qu'on le jelle dans un gouffre. Le mystere ne prouve pas le non-être... Philosophe, dira-i-il, est-ce là ta tolérance? Oui; parce que, si la toglerance est le bien de l'hmmanité, ses bornes doivent se trourer ou ce bien n'exisic plus. Ne sont-elles point assez reculécs, quand on les pose devant le ncant, et les maux épourantables qui l'environnent? 


\title{
34 LA COCHLYOPÉRE.
}

\section{ARTICLE III.}

\author{
$C O R P S$.
}

UN escargot à qui l'on coupe la tête, jette toujours une grande cquantité d'eau. Lorsque cette liqueur ne tombe plus, si l'on met l'escargot sur l'ouverture de sa coquille, dảans une boite ou une caisse vide, il y reste d'ahord immobile; puis, assez ordinairement, il s'y meut et s'y colle, en moins de vingt-quatre heures. Il peut vivre, dans cetétat, un ou plusieurs mois. Cependant il finit toujours par mourir. J'en ai vu qui ont ainsi vécu une année en tière, et qui, ayant été décapités un peu avant le printems, ont été par conséquent une année sans tête, et dixhuit mois sans boire ni manger. Quelque étonnante que soit celte observation, il 


\section{IA COCHLIOPERIE. 35}

n'y a point eu d'erreur de ma part, puisque leur coquille portait la date de leur. exécution.

Lorsqu'on ôte un escargot de la place où il a collé sa coquille, après l'y aroir laissé un mois ou six semaines; on remarque, sur-tout en hivèr, que l'animal s'Gst enfermé dans sa maison, sous une espèce de toile sèche et blanchâtre, faite de sa bave, à une profondeur arbitraire. Si l'on brise cette enveloppe, avec une épingle, on voit les chairs du testacée dans un bel état de fraîcheur. Quand la toile est brunc ou moire, on trouve presque toujours l'animal mort, et dans un commencement de putréfaction. Ainsi, par la seule inspection de cette toile, on peut connaître, en général, si l'escargot est mort ou vivant, sans qu'il soit néces-saire de la déchirer. S’il y a quelques exceptions pour les morts, elles proviennent, soit de la position de la coquille, 


\section{LA COCHLIOPERIE.}

soit du nombre des enreloppes, car il y en a quelquelois deux ou trois l'me sur l'autre.

Quelle que puisse être la durée de la vie d'un escargot decapité, et mis dans une caisse ride, il est facile de se convaincre que sa tele ne se reproduit jamais. Il suffit pour cela de le roir sortir de sa coquille, tous les mois, ou tous les deux mois. Si le reptile n'en sort pas naturellement, on peut l'y contraindre de deux manieres : I". pendant l'hivèr, en l'exposant au suleil, ou à la chaleur douce d'un feu de chemines, ¿̀ environ un pied de distance de la llamme; $2^{\circ}$. dans toutes les saisons, en le louchant, arec la pointe d'une epingle, autour ducollier, ou des chairs inuqueuses qui recouvrent son corps, principalement dans les deus angles du trou de la coquille.

Celte seconde maniere raut mieux (quo la précedente. Mais il faut y aller ares 


\section{A COCHLIOPÉRIE. $3 \%$}

anouceur, et prendre garde de rien brusquer. Lorsque l'escargot est une fois rebuté, il s'enfonce de plus en plus dans sa retraite, et on le tuerait plutôt que de l'en faire sortir. Alors il faut changer de procédé. Le seul moyen de le contraindre à se montrer, est de le jeter dans une jatte d'eau pure, d'une chaleur tempérée, ou de faire tomber sur lui, avec un arrosoir, une pluie artificielle. Ces deux méthodes sont infaillibles, particulièrement la première. On doit seulement faire attention qu'elles ne valent rien, quand on veut priver le colimaçon de loute espèce de nourriture, puisqu'on ne peut le mouiller oa le meitre dans l'eau, sans le faire boire. Aussitôt qu'il commence à sortir de sa coquille, on ne peut trop se hâter de le poser sur une table, afin de l'observer dans sa marche. Il n'y a point de tems à perdre, ilu moins si on l'a excité à se mouvoir, 


\section{LA COCIILOPERIE}

par la chaleur, ou par l'attouchement. Souvent l'hermite rentre, après un intervalle fort court, et il est rare cqu'il se remontre deux fois de suite. On peut aussi le tenir à la main, pour lui roir senlement allonger le cou. Il est pourtant préférable de le laisser agir en liberté, parce que l'escargot ne marche point sur son empatenent ou sa plaque inférieure, sans développer sa tête autant qu'elle peut l'être. De quelque manière qu'on lohserve, on ne lui découvrira jamais aucune apparence de tête; on ne verra qu'une pellicule sur l'incision, avec la peau du cou un peu repliée en rondeur sur cette pellicule.

Ainsi, Voltaire n'était pas fondé it dire qu'un escargot ne peut virre sans ête, ni sans cerrelie. Le corps de cet animal, comme je l'ai dit, peut vivre sans tête une année entière, renfermé dans une caisse vide, quoique cela stit 


\section{TA COCHLIOPERIE. 39}

sans exemple parmi ious les êtres animés.

Dès qu'un escargot se meurt, il répand ardinairement une liqueur brune, qui sort de sa coquille, lorsqu'elle se trouve sur un plan horisontal, et qu'elle n'est fermée que par une seule toile. Le lendemain, si on le perce dans son collier, avec une grosse épingle ou un clou, on le tire facilement de sa retraite. Il n'y tient que par juxta-position, et par une petite membrane, qui l'attache à la columelle ou axe de la dernière spirale de son coquillage. Pour s'en convaincre, il faut découvrir un escargot, mort ou en vie, depuis la partie extéricure de la pointe, jusque rers l'orifice de la dernière spirale. On verra que lanimal ne tient en aucune façon à sa coquille, du côté de la queue. Mais si m le dégage doucement de la columello, -n de l'aye des spirales, autour duruel it 
est roulé sur lui-même, en commenças. par le collier et la tête, on s'apercevra qu'me membrane blanche l'attache à l'axe de la dernière ou avant-dernière spirale. Ceux qui ont prétendu le contraire, ont mal observé cet endroit ; ils ont certainement déchiré le ligament dz coquillage, en le tirant avec peu de précaution.

Un escargot étant mort, après aroir été décapité, l'eût-il été depuis dix ou douze mois, ce serait en vain quion le tirerait de sa coquille, et qu'on le disséquerait, la loupe sous les yeur, pour chercher intérieurement une reproduction de têle. Il n'y en a point.

Quand il meurt hors de sa coquille, ce qui arrive quelquefois, si on lui prend la peau clu cou et celle de la gorge, avec deux pinces, et qu'on les tire en sens contraire; on découvre, an-deli de l'emplacement de ses anciemnes cornes, une 
petite poche tendineuse, dans lacuelle on pourrait insérer un pois. C'est dans cette poche que l'escargot replie son capuchon, et qu'il cache sa tête, quand il en a une. Dès-lors qu'on ne troure rien dans cette poche, c'est que l'animal n’a point régénéré sa tête.

Un escargot, qui ne meuri pas sur un plan horisontal, ne laisse aucun indice de son décès, parce que l'eau qu’il jette reste dans la coquille. On ne peut en être instruit, cqu'au bout de plusieurs jours, par l'odeur cadavéreusequi s'exhale de son corps. Dans cette position, il n'es: pas toujours facile de reconnaître le défunt, au milieu des escargots acépha. les qui l'environnent. On ne doit pour. lant pas négliger de le retirer du troupeau; il serait dans le cas d'infecter tout un appartement. Lorsquion l'a déconvert, il est quelquefois desseché, d'aurefois il est rempli de vers rongeurs, ef 


\section{L A COCHLIOPÉRIE。}

d'autrefois il nage dans une eau putride qui prorient de sa queue ou de ses intestins.

Beaucoup de personnes croient que la chair de ce testacée se tranforme toujours en cette eau infecte; c'est une erreur. La chair de l'escargot est trèsferme, très-coriace. Elle se sèche, se corrompt ou se putréfie, de la même manière que celle de tous les autres animaux, selon les circonstances qui précèdent ou qui accompagnent sa mort. Le roi David, parlant de ses ennemis, a donc eu tort de crier au Seigneur, dans le Pseaume LVIII, verset 9, texte héhreu : "Que chacun d'eux s'en aille, " comme un colimaçon (schablul) qui "se fond!" Dieu qui sait que les colimaçons ne se fondent point, a dû être fort étonné de cetle sainte et charitable prière. Je pense toutefois qu'il n'aura point tenu compte de celte pécadille, 


\section{LA COCHLIOPERIE.}

à celui qui dit au Pseaume XCVII, verset 5 , que « les montagnes se fondent " comme de la cire, à cause de la pré"sence de Dieu; " ce qui passe la plaisanterie (2).

(2) Je suis ici le texle hébreu, et non la Vulgate latine dont S. Jérôme est l'auteur, parce qu'il a changé une infinité de passages de la Bible originale.

Celte Vulgate dit, au Pseaume LVII, verset 9: Sicut cera, quce fluit, auferentur; "Ils 2) seront détruits, comme la cire que la cha» leur fait couler! „ Elle dit, au Pseaume XCVI, rerset 5: Montes, sicut cera fluxerunt à facie Domini; "Les montagnes se sont fon》) dues comme de la cire, à l'aspect du Sei») gneur.» On roit qu'elle ne se fait pas grand scrupule de changer, selon ses rues, ou son caprice, l'idée, la chose, le tems, et jusqu'au numéro des Pseaumes.

Ces licences, qui sont très-multipliées dans la Vulgate, l'ont fait comparer à un ruisscars 


\section{it LA COCHLTOPERIE.}

trouble d'inscntions humaines. Je ne décide point de quel côté est le ruisseau trouble; mais je dis que la Bible hebraique, elant réputéc sucrée, derait nous être transmise telle qu'elle a exé écrite par les auteurs originanx. Notre $\checkmark$ uggate, au contraire, est si peu d'accord avec le iexte primitil, qu il est presque impossible de wettre le doigl destus a sne courris nno trache. 


\section{A R T I C L E IV.}

\section{COQUILLE.}

$E_{N}$ hivèr, la coquille des escargots est ordinairement fermée d'un opercule calcaire, ou croûte blanche, épaisse et dure, qqu'il est bon de savoir enlever, sans blesser l'animal, ni dégrader sa demeure. A cet effet, on gratte, avec un canif, cette porte artificielle, du côté du corps de la coquille, de manière à y pratiquer une pelite fente, depuis l'angle intérieur jusqu'i l'angle extérieur. On introduit la pointe du canif dans cette fente, à l'angle intérieur ou du centre, on souleve doucement la croûte, et el'e se dét che des bords de l'orifice, romme s'il n'existait entrenx ancune expece d'adhérence. On

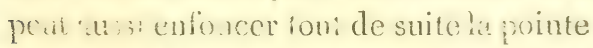
t'un clou à l'angle interieur, et soulerer 
cette croute; par ce procédé, cn la detache également toute entière, çnoiqque. pour l'ordinaire, elle se rompe en plusieurs pièces.

Cette première cloison enlevée, on en ôte une seconde, qui est une simple pellicule transparente, marquée d'une tache blanche, à l'endroit cqui recourre l'anus des escargots. Il faut la déchirer avec précaution, parce qu’elle affleure quelquefois les chairs de l'animal. Ceperdant on en trouve le plus souvent deux ou trois les unes au-clessus des autres.

Quand l'orifice de la cocquille est ourert et bien net, on en fait sortir l'escargot, par les procédés que j’ai enseignés. Enstiite on le mutile, comme on le juge convenablo; ê l'on écrit, avec de l'encre, sur le dos de la coquille, l’opération quon a faite, ainsi que la date. Cette inscription ne s'efface point, lors que les escargots passent lez uns sur les 


\section{LA COCHCYOPÉRIE. 47}

auires; elle ne s'efface pas mème à la rosée, ni à la pluie, si ce n'est après un tems considérable.

Pour peu qu'on ait vu et touché des escargots, on doit aroir remarqué que cet animal reconslruit sa coquille, soit qu'elle ait été brisée du cóté de l'ouverture, soit qu’elle ait été enlevée dans quelque partie du corps ou de la quene. Cette reproduction pierreuse s'opère par la transpiration du testacée, arec une proinptitude élonnante, dans la bellesaison. Cela me fait présumer que, si l'on pouvait conserver la vie à un escargot, dans un lieu convenable, et l'y nourrir quelque tems, entièrement dégagé de sa coquille; cet animal se-formerait une nouvelle de. meure, sans autre secours que la substance risqueuse qu'il ferait sortir de toutes les parlies de son corps. Cette expérience mérite d’étre tentée par quelque habile naturaliste, quoiqu'elle soit plus curieuse qu'utile. Au reste, qu'elle deman- 


\section{S LA COCHLIOPÉRIE.}

de peu ou point de précaution, il est certain que l'on fait reproduire à l'escaryot, toutes les parties de sa coquille successivement, depuis la première spirale jusqu'à la dernière, ou depnis la pointe jusqu'à l'ouverture. Les reproductions de ces parties, à la pointe et au corps de la coquille, sont manifestes, en moins de vingt-quatre heures. Elles parviennent à une consistance pierreuse assez forte, en douze ou quinze jours, sur-tout quand l'animal prend de la nourriture. Celles de l'orifice sont plus lentes, parce qu'il est nécessaire que l'escargot se gonfle, ou se mette en mourement, pour rétablir sa demeure, et qu'en cet endroit il la rétablit ordinairement à plusieurs reprises. Néanmoins elles peuvent s'effectuer presqu'aussi rapidement que les autres, quand la brisure n'est pas large, et que le collier est un peu découvert, le testacée se Irouvant ému par le contact de l'air ambiant. 


\section{A RT ICLE V.}

'TMS ILE PLUS PROPRE AUX OPÉRATIONS.

LA saison la plus arantageuse aux cxpériences que l’on peut faire sur les escargots, est le commencement du printenıs, quelques jours après que ces animaux sont sortis de terre, pour se nourrir re gazon et de rosée. L'été est trop chaud, ou trop près de l'hivèr ; l'automne est trop sec et trop court; l'hivèr est trop froid.

Comme il est d'un Auteur raisonnable de ne jamais rien écrire qui ne soit de quelque utilité, je passerai sous silence toutes lesopérations que j'ai faites durant ces trois dernieres saisons. Celles dont je parlerai dans la suite de cet ouvrage, derront ètre rapportées à la première, 5 


\section{5o LA COCHLTOPÉRIE.}

parce qu'elles ont toutes été entreprises au commencement du printems, c'està-dire, dans les deux mois de la germination des plantes et des reproductions de la Nature. Cela ne s'oppose point à ce que les persommes qui roudront faire des expériences sur ces reptiles, pour leursatisfaction particulière, ne les entreprennent à des ćpoques quelconques. Il suffit qu'elles soient averties, qu'en général, elles parvicndront rarement ì d'autres résultats que ceux dont j’ai parlé dans les articles précédens; leurs expériences du moins resteront toujours imparfaites.

S'il faut en croire l'opinion vulgaire, le commencement du printems est aussi l'époque où les escargots sont bons à fricasser. Les uns prétendent qu'ils sont excellens jusqu'au moment oì ils s'accouplent, vers le milieu ou la fin de cette saison; les autres assurent qu'ils ne sont nlus bons à manger, dès qu'ils ont ietri 


\section{A COCHLIOPERIE. 5I}

leur opercule, et pris de la nourriture. Il ne faut pas disputer des goûts. Ce proverbe, quoique trivial, est bien ici à sa place. Je n'ai jamais pu manger qu'un seul escargot; et pour faire connaître combien le gibier m’a plu, je dirai que je n'ai point eu le courage de l'avaler.

Il y a pourtant des contrées, telles que la Silésie, le Brabant, le pays de Liège, la Suisse, l'Italie, et plusieurs départemens de la France, où ils sont aussi estimés que les huitres. On m'a assure que, dans les environs de la Rochelle, on les fait parquer, en les mettant les uns au-dessus des autres, par couches, entre chacune desquelles on étend de li mousse et d'autres plantes. Cet expédient était inconnu aux Grecs et aux Romains, qui étaient assurément d'aussi grands mangeurs d'escargots que nos modernes. Pline l'ancien, qui fait mention de leur gुoût pour ces reptiles, dans son Histoire 


\section{LA COCHLIOPERIE.}

Naturelle $\left({ }^{*}\right)$, dit que les meilleurs escargots leur venaient re l'íle d'Astypalée, une des Cyclades, daus la mèr Egée ou l'Archipel. Selon le meme auteur $\left(^{* *}\right)$, ils en tiraient aussi du tersitoire Ie Réate, dans la Sabine, cn Italie; de l'Illyrie, contréc d'Europe, le lonog de la mèr ou du golfe Adriatique; de divers pays de l'Africque, et plus particulièrement de Solite, dans la Mauritanie Tangitane, aujourd'hui le royaume d'Alger. Ce Naturaliste dit encore (***) qu ils faisaient beaucoup de cas des escargots de Sicile, de ceux des îles Baléares, aujourd'hui Majorque et Minorque, et de ceux de l'ile de Caprée, aujourd'hui Capri, sur la cồe du royaume de Naples,

$\left.{ }^{\star}\right)$ Ilistoire Naturelle de Pline, livre VIII, chapilre 39 .

$\left.{ }^{* \star}\right)$ Ibidem, livre IX, chapitre 59.

${ }^{\star * *}$ Ibidem, livre $\mathrm{XXX}$, chapitre 6 . 


\section{IA COCHLIOPERIE. 5 S}

fle fameuse pour avoir été le séjour de l'infâme et cruel Tibère.

L'usage des Romains était d'enfermer toutes ces différentes espèces d'escargots lans des garennes et des réservoirs, où ils les engraissaient avec du vin cuit, de la farine, et d'autres ingrédiens. Cette méthode valait mieux que celle des Rochelois, en ce qu'elle laissait à ces animaux la faculté de se reproduire. Malgré cette précaution, qui derait toujours procurer aux anciens une grande abondance de colimaçons, ils ne laissèrent pas de manger ceuxmêmes dont ils tiraient leur belle pourpre. Athénée le témoigne, dans son Banquet des Savans (*). Il ne faut pas douter qua ce ne soit leur gourmandise qui en ait détruit, ou presqu'entierement détruit l’espèce, ei qui ait causé la períe

${ }^{*}$ ). Le Banquet des Savans, par Athénée, livre III, arlicle des Coquillages. 


\section{5/ LA COCHLIOPÉRIE.}

de la plus magnifique couleur violell: et rouge, qui ait existé dans le monde.

Sous un autre rapport, les fricassées d'escargots sont très-célèbres dans les annales des empires. L'histoire de la guerre de Jugurtha, par Salluste, nous apprend qu'une fricassée d'escargots fut cause que ce roi de Numidie perdit ses immenses trésors, ses armées, sa couronne et la vie. On lit, dans la Bibliothèque Anglaise (*), que, lorsque les Espagnols firent la concquête de l'Amérique, ces brigands ayant trouré, près de Sainte-Marthe, des paniers où les habitans avaient enfermé leurs denrées, lesquelles consistaient en colimaçons et sauterelles; ils eurent tant de mépris pour cette nation, quils fondèrent lidessus le droit qui rendait les Améri-

$\left.1^{*}\right)$ Bibliotheque Anglaise, tome XIII, pare 2 , arlicle 3 . 


\section{LA COCHLIOPERIE.}

cains esclares des Espagnols, et celui qu'ils roulaient avoir de les massacrer. Selon d'Herbelot, dans sa Bibliothèqu' Orientale, au mot Kessariah, les Mahométans d'Alger ont une opinion bien difiérente : ils prétendent que les escargots sont saints, comme les cochons l'étaient dans l'ille de Crète; mais, par cette raison, ils s'abstiennent d'en manger, s'imaginant que ceux qui s'en nourrissent commettent un très-grand crime. Il semble, en vérité, que ces paures animaux soient créés pour être les jouets des préjugés des hommes. Les Juils, par nne autre bizarrerie, ne prétencieni-ils ras que les escargots sont aussi impura que les cochons, et les conhons ansi impurs que les escargots? Mo:se, dans le Lévilique, texte lebreu, chapitre XI, verset.3 29 et 30 , leur déferil crpressíment de manrer des escargols, diant: "Caci rous sora aussi inmonde, cntws 


\section{LA COCHLIOPERIE.}

"les reptiles qui rampent sur la terre, "savoir : la belette," (qui, par pa"renthese, ne rampe pas, ) "la souris," "(quine rampe pas davantage, « la tor" tue," (qui traîne sa maison sans ram" per, ) " chacune selon leur espèce; le " Lérisson, le crocodile, le lézard, " (qui " ne sont point des reptiles qui rampent, ) " "le limaçon (cometh), et la taupe," " ( qui n'est point encore un reptile qui "rampe.) (3).

(5) Non-seulement la Vulgale a changé l'ortre el le nom de presque tous ces animaux, mais encore le texte indicalif. Au lieu de entre les reptiles qui rampent sur la terre, ello dit entre les animaux qui se remuent sur la terre.

J'ćcris celte nole, aussi bien que la précédenile, pour les personnes peu au fail, qui, ne connaissant point le texte original, me reprocheraicn! suressest d'angir altéré notre Bible. 


\section{LA COCHLIOPÉRIE. 57}

S'il faut en croire le père Sicard, dans les Lettres édifiantes (*), les Juifs n'ont pas tonjours eu cette aversion pour les limaçons, selon leur espèce. On lit, daus une de ses Lettres, que la vallée par oiz les Israëlites passèrent, en fuyant d'Eypte, pour se rendre à la mèr Rouge, " est remplie d'une quantité si prodi" gieuse de giros limaçons, que l'on ne " peut faire un seul pas sans marcher " dessus. Ils sont excellens; et il ne faut " pas douter cque les Israëlites en aient "fait de bons festins, pour ménager " leur bétail. " Le père nous dit peutêtre là une sottise. Toujours doit - on en conclure que la vallée de Gendeli, de Ramlie et de Bedé, en allant du Caire à la mèr Rougre, est le royaume des escargots. S'il y a d'autres pays ou ils soient

(*) Leltres édifiantes, tome V, page 278 , édition de 1780 . 


\section{8}

\section{LA COCHLIOPÉRIL.}

très-abondans, il n'y en a point où on les trouve en aussi grande quantité, ni d'une aussi belle espèce.

Pour en revenir à mon sujet, il est vraisemblable cqu'en Egypte, et dans quelques autres contrées d'une pareille température, toutes les saisons seraient également propres à faire des expériences sur les escargots. Mais, puisque nous n'y sommes point, ce que j’écris sur ces repliles ne doit se rapporter qu'au climal de la France. Lors même que je ne le dirais pas, on reconnaitrait, par la seule réflexion, que les résultats de mes recherches seraient différens, ou éprouveraient quelques modifications, dans des pays où la belle saison aurait plus ou moins de durée. 


\section{A COCHLIOPÉRIE. $5 y$}

\section{A R T I C L E V I.}

NOURRITURE.

Toutes les opérations que j’ai faites sur les escargots n'ayant pas été bornées à leur couper la tête, j’ai cru que ceux de ces animaux à qui je la conservais ne devaient pas être mis dans une caisse vide, puisqu'ils pouvaient manger. En conséquence, j'ai fait construire une longue caisse, dans laquelle j’ai mis de la terre franche, avec un tapis de gazon, qui a été arrosé tous les jours, et renouvelé tous les mois. La partie supérieure de cette caisse avait un tiers de sa largeur convert d'une planche; les deux autres tiers étaient fermés par un petit grillage à charnière. Il est nécessaire que ce grillage soit en fil de fèr : un grillage de laiton pourrait empoisonner l'herbe, ot 
quand il est de fil ou de ficelle, les escargots le mangent.

Cette caisse ayant été placée sur une fenêtre exposée au nord, les escargots s'y trouvèrent aussi fraîchement que cela était nécessaire pour leur conservation. La planche supérieure leur offrait un abri contre les mavais tems; et le grillage laissait introduire, chacque nuit, une rosée qui n'était pas moins salutaire à l'herbe quaux escargots euxmêmes. Indépendamment du gazon qui devait les nourrir, je meitais, assez régulièrement tous les jours, clans cette caisse, des feuilles de rigne, de tilleul, de rosier, de chicorée sauvage, de presque toutes les plantes potagères, et principalement de bryone, que les escargots de igne aiment beaucoup, lorscru'elle est fraiche, jenne et tendre. La bryone on vigne blanche (bryonia seu vilis alba) ist peul-etre plus comme sons le non: 


\section{LA COCHLIOPERIE. 6 :}

de couleuvrée, ou de navet sauvage. On. la troure dans les haies, les bosquets ot les bois; elle s'accroche de branche en branche, comme la vigne; et elle a. des feuilles qui ressemblent beaucoup à celles du lierre, si ce n'est qu'elles ne sont pas aussi épaisses, ni d'un vert aussi foncé.

L'escargot mange cncore de la terre; il s’en nourrit quelquefois jusqu'à satiété. Un jour, ayant trouré, dans les bois, un escargot d'une grosseur prodigieuse. je brisai sa coquille, et je lui fendis le dos dans toute sa longueur, pour en examiner l'organisation. Je ris la poche de son estomac très-cnflée, et pleine de terre pure.

Les personnes qui enformeront des escargots dans une caisse semblable a celle que je viens de décrire, pourront donc leur procurer facilement les clioses nécessaires à la vie, puisque ces animaur 


\section{LA COCHLIOPERIE.}

zirangeront de l'herbe ou de la terre. quand on n'aura rien à leur donner. Ils mangeront aussi du papier mouillé, si l'on reut en mettre dans leur caisse; même de la farine en poudre, ou en pâte liquide, dont ils sont très-friands.

Les divers inconvéniens que présente celle cspèce de cage, ue peuvent malheureusement s'eviter. Si l'escargot se colle sur l'herhe, il en sort quelquefois le vèr de terre commun, nommé le lombric, qui le tue et le ronge, sans quion s'en aperçoive. S'il se colle contre la planche, et que cette planche soit piquée des scarabées, des larres de phalcues, et, en général, des vers rongeurs quion nomme vers tarières, ces insectes ne leur sont pas moins redoutables. Il n'y a que le grand nombre des prisonniers qui puisse consoler un observateur, des pertes produites par ces enmemis parasites, el peut-être aussi nar 


\section{LA COCHLIOPERIE. 63}

le défaut de liberté; car il en meurt con sidérablement.

En hivèr, avant que le froid devienne excessif, on doit mettre la caisse dans une cave; ou bien, répandre les escargots dans un tas de pierres, placé devant un mur, du côté du midi, et jeter, par dessus, quelques pelletées de menue paille et de feuilles mortes. On peut encore les mettre dans une boîte pleine de son, et placer cette boîte à un rezre-chaussée, dans un endroit aéré, où I'on ne fera point de feu. Quant à leur nourriture, durant cette saison, il ne faut pas s'en inquiéter, parce qu'ils ne mangent point. 


\section{b4 LA COCIILIOPERIE。}

\section{A R T I L E VII。}

$E M P A T E M E N T$.

Sr l'on coupe, avec de grands ciseaux: la partie postérieure de l'empatement, ou toute la queue extérieure d'un escargot, il la recourro dans l'espace de six mois. Mais la peau n'en est pas rugeuse ou grenue, comme au reste du corps. Avec beaucoup de tems, il serait possible que cette peau se rétablit dans son état naturel. Néanmoins sa régénération n’a jamais été parfaite dans mes opérations, quoique j’aie eu occasion de garder des escargots mutilés, pendant près de deux ans. Cette recherche, au reste, n'est pas digne d'une application particulière; elle ne peut conduire ì rien, et ne présente qu'un objet de curiosité puérile. 


\section{IA COCHLIOPÉRIE. 65}

\section{ARTICLE VIII.}

$C O U$.

Sr l'on fend le cou d'un escargot, derrière les grandes cornes, c'est-à-dire, si on lui fait, dans cet endroit, une incision transversale, qui lui fende le cou à moitié; la blessure se ferme, et la peau se recolle en moins de deux mois. Quelquefois les grandes cornes en deviennent plus petites; d'autres fois, il n'y en a qu'une seule cqui 'dégénère. J'ai vu un escargot ainsi blessé et guéri, dont une des grandes cornes paraissait à peine, tandis que l'autre était une fois plus longue qu'arant l'opération; ce qui en arait fait une espece d'escargot licorne. 
66 LA COCHLIOPERIL.

\section{A RTICLE IX。}

\section{$F R O N T$.}

UN escargot qui reçoit un coup de ciseaux dans le front, au milieu de l'espace situé entre les deux grandes cornes et les deux perites, n’en éproure aucun accident, et les chairs se recollent en moins de deux mois. Cependant il fut éviter de plonger le coup trop avant. Lorsque la mâchoire supérieure se trouve entièrement séparée du reste de la tête, ou n'y tient que par les joues et les lèvres, elle se corrompt, et cause infailliblement la mort de l'animal. 


\section{LA COCHLIOPERTE. 67}

\section{A RTICLE X.}

\section{CORNES.}

Sr l'on coupe une ou plusieurs cornes, ou toutes les cornes, à un escargot, elles se régénèrent entièrement, dans l'espace de cinq ou six mois; mais elles sont toujours couvertes d'une peau lisse. Pour couper toutes les cornes ì un escargot, on le laisse ramper sur une table, on larrête par la coquille, et on les lui enlève, Thune après l'autre, avec de petits çiseaux, sans la moindre difficulté. A chaque coup de ciseaux, l'animal se replie un peu sur lui-même, et retire les autres cornes. Il faut atlendre qu'il les allonge de nouveán, et l'on ne doit continuer les amputations, que lorsqu'on aperçoit le globe qui est à l'eàtrémité des cornes, bien formé, bien coloré, bien chargé de liqueur. Autre- 


\section{IA COCHLIOPERIE.}

ment on pourrait ne couper que la peau, comme il est arrivé à Voltaire : l'expérience apprend que les cornes, quoiqu'assez bien tendues, ne sont pas toujours remplies de la liqueur noire qui y circule.

Si l'on divise les cornes d'un escargot, dans toute leur longueur, elles se guérissent en deux ou trois mois, et prennent la forme d'un bois de cerf ou de faon. Pour diviser ces cornes, on fait ramper l'escargot sur un petit ruban ou une grosse ficelle, et on l'étrangle vivement près de la tête. Cet étranglement maintient les cornes noires et immobiles. Les quatre cornes étant bien fendues, avec de petits ciseaux, et les parties bien séparées l'une de l'autre, on coupe le nœud du ruban ou de la ficelle, et l'animal commence aussitôt à se retirer dans sa coquille. Il s'y retire lentement, moins peut-être parce qu'il souffre beaucoup, 


\section{LA COGHLTOPÉRIE. 69}

que parce qu'il a été engourdi par la strangulation.

On a beaucoup disserté sur les cornes des escargots. Quelques naturalistes pensent que ce sont des antennes, par le moyen desquelles ces animaux sont prévenus de la rencontre des corps qui se trotivent sur leur passage. Iie plus grand nombre croit que ce sont des yeux, ou des cornes œillées. Fort peu de personnes ont préiendu que ce sont des nez, ou des organes de l'odorat.

Le premier avis est vrai sans conlestation; mais il n'apprend rien. Les cornes des escargots, fussent-elles des nez ou des yeux, ne laisseraient pas d'être des antennes, par la seule raison qu'elles sont des cornes mobiles. Encore est - il plus convenable de dire qu'elles sont des tentacules, ce nom étant spécialement domné aux cornes de certains mollusques, parce qu'elles se tendent, cu pros 


\section{TO LA COCHLIOPÉRIE.}

qu'elles sortent de la tête et y rentrent, à volonté (4).

Le second avis semble pécher contre l'évidence. L'animal ne se détourne point, quand on passe le doigt, ou toute autre chose devant sa tête; et lorsqu'on lui a coupé une corne, il ne paraît nullement appréhender les ciseaux qui vont lui en couper une autre, pourvu qu'on ne touche pas le plan sur lequel il rampe, ou cqu'on n'agite pas trop brusquement l'air qui l'environne.

Quant au troisième aris, on peut trouver extraordinaire que les escargots aient besoin de quatre nez, pour manger de l'herbe ou de la terre, et que ces quatre nez soient dans une direction opposée à leur bouche, ce qui les rend absolument

(4) On donne le nom de mollusques à tous les animaux qui n'ont qu'une chair molle, sans os. Tous les molhusques n'ont pas des cornes. 


\section{A COCHLIOPÉRIE. 71}

inutiles. Cependant il est incontestable que ces animaux ont un odorat exquis, lor's même qu'ils sont renfermés dans leur coquille. Je les ai vu collés, plusieurs jours et plusieurs nuits de suite, contre leur caisse, chacun à la même place; je leur ai donné, un soir, des plantes fraîches; et le lendemain, à la pointe du jour, ils étaient tous, ou presque tous, à les dévorer. Un savant moderne, après avoir fait sans doute une pareille observation, a dit que le nez des escargots est répandu dans toute leur peau. Ce mot résoud un grand problême. Je soupçonne toutefois que cet auteur, quoique plein de mérite, est un peu dans le cas du bûcheron qui jette le manche après la coignée. Oì est counc le nez des escargots? je l'ignore. Je ne conçois pas un méchanisme organique qui ne clomne aucune prise à mes sens, et l'on vient de m'apprendrequ'il est bon quelquefois de. sacrifier an silence. 


\section{IA COCHLIOPERTE.}

Je ne me rappelle pas qu'on ait dit que les cornes des escargots sont des oreilles. Si je pouvais avoir un sentiment sur ce sujet, je ne sais trop si je n adopterais point cette dernière idée. J'ai plusieurs fois remarqué, en m'approchant d'un escargot, que le bruit de mes pieds contre l'herbe, lui causait de la frayeur, et le faisait rentrer dans sa coquille. M'entendait - il par les cornes, ou par quelquautre partie du corps? c'est ce qu'il ne m'est pas possible d'affirmer. Je ferai seulement remarquer, qu'il n’y cu a aucune autre qui soil plus propre à cet usage, et qui réponde mieux au plau ordinaire de la nature.

Quoi qu'ilen soit, je vais proposer trois moyens de s'assurer si les cornes des escargots sont des lunetles d'approche: des tuyaux olfactoires, des cornets aconstiques, ou de simples antennes.

Faites sortir un escargol de sa coquille, 


\section{LA C OCHLIOPERIE. 75}

et laissez-le ramper sur une table, dans un lieu sombre. Prenez une paire de petits ciseaux frottés de quelque matièrt: phosphorique; coupez-lui une pelitc corne, et retirez-vous. Un instant après, coupez-lui l'autre petite corne, et retirezvous encore. Faites la même chose aux deux grandes cornes; et soyez persuadé que, si l'animal n'est point effrayé à l'approche des ciseaux, après une ou deux cornes coupées, c'est qu'il ne voit point par les cornes. Cette vérité serait incontestable, lors même qu'on le supposerait nyctalope, ou royant mieux la nuit que le jour, puisque rien ne s'oppose à ce qu'on le mette dans une obscurite plus ou moins profonde.

A la suite de cette première expérience, failes - en une seconde, sur un autre escargot, en vous servant de ciseaux frottés d'alkali volatil, drogue qui - une odeur extrèmement forte. Vous 


\subsection{LA COCHLTOPERIE.}

conclurez, par une même analogie, que les cornes de cet animal lui servent ou ne lui servent point de nez.

Enfin tentez une roisieme experience sur un nouvel escargot, en lui compant chaque conne, immediatenent après un petit roulement, fait par une autre pera somne, sur un tambour, ou avec une somnette. Si l'animal se retire au premier bruil, c'est une preure qu'il l'entend; et il sera nécessaire de l'y accoutumer, avant de commencer l'expérience. Il ponrraii aussi l'entendre, et ne pas sc retirer, ce bruit ne lui ayant encore fait aucun mal. Supposant que l'escargol n’en soit point ou n'en soit plus ému, s’il se retire, apris une ou deux cornes coupées, c'est qu'il entend certainement le bruit, par quelque endroit que ce puisse être; s'il ne se retire plus, pendant le roulement, lorsque ses quatre corncs seront coupées, c'est qu'il entendait par les cornes; et s'il 


\section{IA COCIIIIOPERTE. 75}

se retire, après cette mutilation, c'est qu'il entend par la racine des cornes, ou par quelquáatre endroit inconnu. Dans ne indecision, les deux premières expériences sur les yeur et les nez, n’ayant point eu de succes, l'anatomie comparée doit autoriser à dire que les cornes des escargots sont des oreilles.

Mais, dans les trois erpériences, si l'animal demeure constamment immobile, à l'approche des ciseaux, ou immédiatement arant chaque amputation, il n’y a plus à discuter : les cornes des escargots sunt de simples antemnes; ct nos petits enfans peuvent continuer de chanter leur belle romance:

\section{Colimaçon borgne,}

Montre-moi tes cornes.

Si tu ne me les montres, je le dirai ì ton perre,

A ta mère,

Qui cueillent des roses,

Dans la fosse. 
-6 LA COCHLIOPERIL.

\section{A RTICIE XI.}

$$
C R A I E \text {. }
$$

T.E crine ou le sommet de la lête d'ur: *scargot ćland coupé rapidement, arec de rands ciscaux, de maniere que les Acux grandes cornes soient enlerées d'um seul cou, meme, si l'on veut, les quatre cornes et une portion de la mâchoire; loule la parie amputée se régénère en six ou huit mois. Lopération dont il s'agit ici est cilfficile à exéculer. On ne pent ta bien faire quen tenant l'animal d'une main, lorsqu'il est liors de sa coquille, afin de le placer commodénent sous les ciscanx qui doirent le muiler. Elle demande beancoup d'arlresse, ot l'on ne reussit pas funjours comme on le roum drait. Ohon-Frederic Müler on a eu l'idée avant moi, daus les expériencen 


\section{LA COCHLIOPERIE.}

qu'il a faites, en I768. Mais il cst étonnzunt que cet auleur ait imaginé de dire, que des escargots ainsi tronqqués, élaient des escargols décapitéz, et cu'il ait prétendu domner les résullats de celte mutilation, comme un témoignago de la fameuse assertion de Spallanzani. Cot étrange paradose se trouve au tome socond de son Termium lerrestitum et fiuriatiliam, et dans le Joumal de Physique, au tome de l'année 1770 , seconde partie. Un escargot est riccapilé, loran qu'on lui coupe le con, dans me direotion perpendiculate à l'axe de l'empan. rement ou du tronc, el non lorscruon hi emporte diaronalement une prutie de Ia tête, ce qui ne le prim pas de lous les organes quille rontrine. Mulle? non-

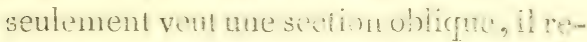
commande en ore, pour dres asame du succès, de prente sorde d'ealaner lo portie antericure de lempatement. ETh 


\section{LA GOCHLTOPÉRIE.}

pareille leçon prouse peu de raisomement ou de honne foi : la mutilation présente n'est qu'une scalpation.

Si celte section du crâne n'a pu me đommer la même satisfaction qu'à Muiiler, clle m'a mis dans le cas d'apercevoir comment les escargots avalent leur nourriture. Ces animaux la prennent avec leurs lèrres; ils la coupent avec un osselet zongếtre et crèielé, qu'ils ont à la mâchoire sipérieure; ct ils poussent, du fond de leur gorge, une langue on forme de cuillèr, qui s'en saisit, et la porte à l'orifice de l'oesophage, dont le mouvement vermiculaire la fait passer dans l'estonac, de même que si ello y était introduite par aspiration. Cette action de la Nature se roit, comme au travers lane vitre, dans la tête des escargots dont on a enlevé la partie supérieure, parce que celle partie se trouve recouverle k'uno pellicule extrêmement mince. 


\section{COCHLIOPÉRTE.}

Une épreuse, qui mérile d'ètre connue, a donné lieu à cette obserration. J'ai voulu saroir si les escargots mangent de la viande. Je leur présentai d'abord du boul bouilli ; ils en mangèrent, mais en fort pelite quantité. Je leur offris ensuite du reau cru ; cotte viande leur plut davantage. Un de ceux qui araient le crâne enlevé, s'étant approché pour s'en nourrir, je l'examinai arec une grande attention, et c'est alors que je m'aperçus comment s'opère, dans ces animaux, l'acte de la déglutition.

Quant à lobjet de cette épreuve, it consistait ì m'assurer si l'on derait ajouter quclque confiance à une anecdote que j'arais lue, dans wn anteur dont le non m'est échnpé, aussi bien que le titre de son ourrage. Cet auteur rapuorte, d'après le président de Thon, que, pondant les gueres de relizions excicics par le payn: Pie IV, il n'y ent point de cruantes qu'on 
n'exerçât confre de malheureux paysans vaudois, qui risnient tranquilles, dans les vallées de Luzerne, d'Angrone, de Pérouse el de Saint-Martin, en Piémont. Puis, citant un trait de barbarie qui lait fremir, et qu'il dit avoir eté rapporte au président de Thou, par des personnes dignes de foi, il ajoute rue cquelques soldats calluliques, avant pris un vaudois, Ázé de suirante ans, le lièrent tout nu sue tu banc, lui onvrient le nombri!, ey mirent un escargot, qua le fit momir hnlement, en lui rongcant jeu à peu les entraill's. L'espéricance que je venais de live, sans conímer absolument cefie anectole, y donnat une espece de rraisemblance; cependantil est bon de savoir, gue le président de Thou ne dit point ce que notre anten lui atribue. De Thou, Jans son Histoire latine universelle $\left(^{*}\right)$,

(*) Jacobi-Augusti Thuani, historiarum sui 


\section{I COCMTIOPLRTE. SI}

dit que ce rieillard fut tué par un $E$ sm carbol, ou scarabée stercoraire, vulgairement nommé Fouille-merde; ce sera, si l'on rout, par un Dcrmestes, espèce de scarabée dissecqueur, qui se nourrit d'ordure et de charogne. Voici son texte: Hominis LX circiter annos nati manibus post tergum revinctis ad scammum alligati, zumbilico scarabaum testa lechum admovent; qui fudicare ac rodere munquam. clesiit, donec in ejus alvimm penelravit, et ila miserum captirum truculento el inauclito morlis gencre enecavit. "Ayant pris " un homme âgé d'enriron soirante ans, " ils lui livent les mains derrière le dos, "l'attacherent sur un banc, et lui milent " sur le nombri!, win escariot, courert " d’une écaille, (ou qu'ils cour rirent d'un " tesson, ) lequel ne cessa de foniller et

temporis, tom.If, ]ib. XXVII, am, CH I:LX, art. X, pag. 92. Londini, 1755. 


\section{LA COCHLIOPERIL.}

" de ronger, jusru'à ce yu'il fut entré $\Rightarrow$ dans son ventre, et eut fait mourir w " malheurcux prisomier, pir ats genre " de supplice aussi cruel qu'inoui. "

Que crlte lausse cilation soil me mauraise traduction, ou une négligence iypographicue, elle m'a fait connainc le systême déglutitil des escargots; cetie connai:sance m'a fiat peuser qu'un colimaçon décapité pent prendre une nouriture liquide; et ce sourcon, qui s'est ironwe fondé, m’a enfin devoile le grand phenomène qui fera le sujet de l'article suivant. C'est ainsi, dit Rémmur, que la "fortune a part aus découvertes d'his" coirematurethe, comme elle en a aux dé"s contertes de tur lis antres gemres $\left(^{*}\right)$."

(*) Mŕmoires ponr servir à l'histoire des Insectes, tome 1, Mémoire I. 


\section{LA COCHLIOPÉRIE. 83}

\section{A R T I C L E XII.}

\section{REPRODUCTION}

DE LA TEETE DES ESCARGOTS.

Dès que j’eus sonpçonné que les escargots peurent se nourrir, par succion ou autrement, après aroir été clécapiés ; je ne tardai pas à conclure qu'ils devaient nécessairement reproduire leur tête, au bout d'in tems quelconque, sclon le systême général de leur organisation ou constitution.

J'ai mis, sur mon pré artificiel, des escargots nouvellement décrpités, avec ceux qui avaient leur téte, el qui me servaient pour l'autres experiences. Nonseulement je l's ai apercus manger quelquefois de la terret des racines d'herhes, mais je leur ai ru jeter des excrémens, en pelite squantité, tout le tems 


\section{I IA COCHLTOPLRIE.}

qu'ils ont élé dans la caisse. La raison qui m’a empêché de les roir manger plus souvent, c’est qu’en général ces animaux ne se promenent et ne se mettent à table que dans les ténebres. S'ils ne sont pas lucifuges, ils sont du moins noctambules, ce qui revient à peu près an mème. Et puis, il faut être prévenu que, lorsqu'un escargol parait fort tranquille surl'herbe, il est sourent occupé à lécher et ponsper de la terre, ce dont on s'aperçoit d'autant plus difficilement, qu'il prend cette nourriture sans sortir de sa coquille.

Ce premier essai m'ayant réussi, comme je m’y étais attendu, il me fut démontré que les escarrots sans téle, qui araient été mis dans une caisse vide, y étaientmorts délaim, plutôt que des suites de leur decollation. Je domai done tous mes suins à mes nouveaux reclus, dans l'espérance de leur roir repousser des têtes. Au bout des six premiers mois de 


\section{I.A COCHLIOPÉRTE. 85}

Labelle saison, j’ai vu avec la plus grande satisfaction qu'elles commençzient ì se reproduire. Cela n'a persuadé que toutes les régénérations des escargo!s, sans aucune exception, ne peurent aroir lieu qu'à l'aide des alimens, comme les plantes ne peurent croître, q̧ue par l'intus-susception des molécules nuiritives de la terre et de l'ezu. Je me suis ensuile plẹinement convaincu de celte rérité, en examinant de tems à autre mes prisonniers, dans le cours de l'hiver suirant. Il est certain que, durant toute celte saison, les reproductions ont été universellement suspendues.

Je me proposais de continuer à nourrie mes escargots, jusqu'i ce que les régénérations fussent compleites; mais ils moururent un jeu avant le printems, sans qu'il m’en restât un seul, même de cenz qui araient leur tête. La cause de cet accident ne m’a pas étć lacile à décou- 
vrir; il est vraisemblable que la température du lieu ne leur convenait plus : je les avais mis dans une cave.

Affligé, comme je derais l'ètre, de ce malheur, je n'eus pas le courage de continuer plus long-tems ces sortes d'cxpériences. Je les suspendis, autant pour me procurer quelque délassement, que pour ne pas être distraii daus d'autres travaux auxquels j’étais livré, et qui demandaient toute l'application de mon esprit.

Une année ensuite, je renouvelai les décapitations, mais sans me soumettre à aucun asulijétis.ement, ni exposer les escargots à aucune cause éirangère de mortalité.

Ayant coupé la tête à deux cenis de ces animanx, je les jelai dans un bosquet hunide, situcé à l'extrémilé d'un jardin, afin qu'ils trourassent plus facilement *outos les cejeces de nourrilures propres 


\section{LA COCHLTOPERIE. 87}

à être avalées, sans le secours de la bouche, ni de la langue. Car c'est en cela seul que consiste le succès de l'expérience, son mystère, et sa grande difficulté. Vers la fin de la belle saison, j’ai vu, à tous les escargots que j'ai pu retrouver, une nouvelle tête, assez ressemblante à un grain de café. Cette tête avait quatre pe¿ites cornes, une bouche et des lèrres; les fraises antérieures de l'empatement élaient Jien reformées. A la fin de l'étć suivant, les têles furent parfaitement reproduites, si ce n'est que la peau en était lisse ou cicatrisée, de même qu’aux amputations partielles.

Par conséquent il faul environ deux années, pour qu'un escargol reproduise sa têle, quand on la lui a coupée avec toutes les précautions dont j’ai parlé au commencement de cet ouvrage. Dans les pays où l'hivèr n'est point assez froid pour faire rentrer les colimaçons au fonct 
88 LA COCHLIOPERTE.

de leur coquille, il ne faudrait vraisemblablement qu'me année, puiscgue les deux ans que dure la reproduction de leur tête, dans noire climat, ne sont antre chose que deux printems el deux étés.

Conchons que Spallanzani ue s'est point trompé, et n'a trompé personne, quand il a dit avoir vu celle reproduction au bout d'un tems indéterminé qu'il semble porter juscru'it deus ans. Il n'est pas fort aisé de comprendre comment la découverte de ce Naturaliste a eté mise su rang des fables, par tous ceus qui ont voulu la vérifier, après aroir lu son Programme. Adanson, entrautres, Adanson, de l'Académie des Sciences de Paris, a décapité, en I 768 et 1769, juscqu'à quinze cents escargots, et n'a janıais pu apercevoir la régénération de leur tête. Cela vient probablement de ce qu'on s'est imaginé cu'il fallait fort peu de tems à ces animaux pour la reproduire, tandis 


\section{A COCHLIOPÉRIE. 89}

que Spallanzani s'exprime assez clairement sur la clurée de cette régénération.

C'est une justice que je lui rends, quoique j’aie composé mon ouvrage, avant d'aroir pu me procurer le sien qui est estrêmement rare. Je ferai même l'aveu que, sur les idées ragues que m'en avaient donné quelques auteurs, je me proposais de l'accuser d'inhabileté clans ses opérations; de démontrer l'impossibilité du phénomène, en deux mois, trois mois, même six mois; et de mattribuer sa propre découverte, qui, sans contredit, est la plus helle qu'on ait jamais faite sur les reproductions animales. Mais dès que j’ai eu lu son Programme, je me suis senti confondu dans ma présomption, et jai reconnu la témérité de mon dessein. Son récit sera toujours très-intelligible et irès-vrai, pour tout homme impartial qui vondra le lire avec quelqne altention. 


\section{9) LA COCHLIOPÉRIE.}

Une seule réflexion serait dans le cas de suspendre l'hommage que je rends à cet illustre observateur. C'est que Valmont de Bomare, et milie personnes avec lui, aient témoigné, à la suite de décollations bien faites, que l’expérience de Spallanzani n'était digne d'occuper ou d'abuser que des enfans; et que Spallanzani', mort en 1799 , c'est-à-dire, trente ans après la censure de Valmont de Bomare, n'ait point attesté, par de nourelles preures ostensibles, „le phénomène cu'il avait ru, comme s'il eût été chimérique, tel qu’il arait cru le voir. Il s'est contenté d'écrire, en 1769 , une lettre à l'un de ses amis, pour lui communiquer ses procédés. Cet ami a fait imprimer la lettre, dans un journal, aussi bien que dans ses propres ouvres; il a répéié les expériences, en faisant usage d'un couteau; il a démontré aux incrédules, un remi-succès, qui ne pou- 


\section{I.A COCHLIOPÉRIE. gI}

vait guère manquer d'aroir lieu; et il n'a converti persomne... Seulement quelques auteurs plus modernes, ayant vu ses demi-preures répandues dans divers ouvrages, ont bien voulu, sans autre examen, lui accorder des régénérations de têtes presqu'entières. Mais Valmont de Bomare, qui vient de mourir de nos jours, en l'année I807, n’est jamais revcnu de son premier sentiment.

Que chacun interprète, comme bon lui semblera, le silence, la conduite, la retraitede Spallanzani! Pour moi, tenant d'une main, mes expériences, bien exécutées, bien étudiées, bien vérifiées; et de l'autre, le seul Programme de Spallanzani, qui me paraît dégagé de toules les fautes et les assertions mensongères de ses rejétitcurs; je dis arec franchise, que je ne peux prétendre qu'au titre de second auteur de la découverte que je viens de publier. Si ma vanité en recueille 
$9^{2}$ LA COCHLIOPÉRIE.

moins de gloire, mon cour en jouira d'une plus douce satisfaction. Juger défavorablement un homme, sans l'entendre, sur l'accusation et l'objection, dans cruelque cas que ce soit; c'est une action indigne, et le fait d'un insensé ou d'un traître, sur-tout quand les choses évidentes font foi pour les choses cachées. 


\section{S U P P L É M E N T}

\section{A LA PREMIÉE PARTIE.}

J'A pensé que la difficulté de se procurer le ProgRAnnE ou Précis des reproductions animales, par Spallanzani, ferait trouver ici arec plaisir, la transcription de ce que cet auteur a dit sur les escargots. On y verra une partie des expériences que j’ai écrites moi-même, avec de petits délails fort intéressans dans lesquels j’ai négligé d'entrer. Il n’y manque absolument qu’un développement des procédés, arec un indice des causes de régénérations.

C'est cette omission qui a trompé un si grand nombre de personnes, et qui a fait débiter tant de fables anx réuéliteurs du Naturaliste italien. Eile les a 


\section{g' LA COCHLIOPÉRIE.}

empêché de voir l'espril, ou la rérité naïve, cachée sous une lettre trompeuse; elle leur a fait croire, leur a même fais dire, que les escargots, sans prendre aucume espèce de nourriture, reproduisent leur tête après qu'elle a été coupée. On ne saurait trop remarquer que, si l'animal ne se mourrit point, la reproduction de sa tête est impossible, et ne peut aroir tout au plus cqun faible commencement, dont le principe est dans le suc des plantes cu'il a mangées avant l'amputation. Qu'ail contraire, si l'animal dérore des feuilles, la régénération n'est plus qu'une illusion des sens, parce qu'un escargot bien décapité, ne saurait prendre pendant longn-tems qu'une nourriture liquide.

Autant que je peux en juger par le récit de Spallanzani, je crois qu'il n'a point connu celte distinction; je crois que des circonstances de tems et de lieux, 


\section{LA COCIILIOPERTE. $9^{5}$}

‘u’il n'a pas su saisir, l'ont servi heureusement dans ses recherches, et l'on conduit au succès, sans qu'il en ait aperç les raisons essentielles. La leclure de son ouvrage, que je vais transcrire sur la traduction française, en comraincra sûrcment tout le monde. Je prériens toutefois que je ne garantis pas le systême anatomique de l'au!eur. Chacun pourra s’éclairer sur cet objet, en disséquant des escargots, après les aroir laissé nourir dans de l'eau fraîche. J'ai aussi redressé le traducteur, dans quelques endroits qu'il n'a point entendus.

Des reproductions de la téte et des autres parties, dans-l'escargot; et cle celle des cornes, dans le limaçun sans coquille, qu'on nomme communément limace; par Spallanzani.

LA tête des escargots est beaucoup plus composée que je ne l'aurais cru. Elle a 


\section{LA COCHLIOPRTR.}

un cerreau, ct ce ccrveau est d'une grosseur considérable: il est formé de deux lobes, par lesquels il est resséré vers le milieu. On roit sortir de la partie inférieure du cerveau, c'est-à-dire, de celle qui est près du corps, deux nerl's trèsapparens, qui ne sont que la moëlle spinale divisée en deux branches. De sa partie supérieure sortent dix autres nerfs, qui se répandent dans toute la tête, el dont quelques-uns se partagent en plusieurs rameaux. Quatre de ces nerfs vont. dans les quatre cornes de l'escargot, et parmi ceux-ci, les deux nerís qui servent anx deux plus grandes cornes sont d'une beauté singulière. C'est à l'extrémité de ces grandes cornes que sont les yeur du testacée. On y décourre distinctement cinc parties : deur tuniques, qui sont l'uvée et l'araihnoide; et trois humeurs, qqui sont, l'aqueuse, la cristalline et la vitrée. 


\section{LA COCHLIOPERIE.}

On observe aussi, dans la tête de l'es. cargot, plusieurs muscles destinés à ses divers mouvemens. Chaque corne est pourvue d'un muscle, par le moyen duquel ce reptile la meut, en avant, en arrière, et la cache à son gré dans sa tête.

L'escargot a non-seulement une bouche, mais des levres, une langue, un palais, un ventricule, des dents, etc.... L'intime el étroite liaison qui règne entre ses dents, fait cqu'elles paraissent n'en former qu'une seule. Leur substance est la même que celle de la corne, dans les autres animaux.

De toutes les parties, si variées critr'elles, dont la téte de l'escargot est. composée, et dont le savant Srvanmerdam nous a domné l'anatomie, laquelle n’a paru très-exacte, je n'en fais commaîIre qu'un tres-petit nombre, en passant. afin que l'on sache combien est admi- 


\section{$9^{8}$ LA COCHLTOPÉRIL。}

rable la reproduction de la tête de l'escargot, quand on réfléchira aux faits que je vais rapporter.

Premierement, l'escargot peut reproduire ses cornes. La manière dont celte régénération se fait, différant beaucoup des autres régénérations, elle nous fournit de nouvelles lumières sur la théorio des reproductions animales.

Dans les autres animaux, on voit paraître, au commencement de cette sorte de reproduction, une petite corne, ou un petit tubercule, dont la base est incomparabiement moins étendue que celle qui reste au tronc, et ce n'est qu'après un long espace de tems quie cette grande différence disparaît. C'est toute autre chose, dans la reproduction des cornes retranchées de l'escargot. Le tronçon même s'arrondit, en formant un bouton, d'une couleur beaucoup plus foncée que le reste de la corne. Ce petit bouton 


\section{LA COCHIIOPERIE. 99}

s'élève, sa couleur devient plus vive, et à son extrémilé, du moins aux grandes cornes, paraît un petit point noir, qui est l'œil de l'escargot. Cependarut la corne mutilée s'allonge, et au bout de quelques mois, elle égale l'autre corne, si celleci n'a pas été coupée. Cette régénération n'est donc qu'une croissance du tronçon. Quant à la couleur de la partie reproduite, elle est tonjours plus claire, dans les premiers tems, que celle de la partie préexistante. C'est d'une manière semblable que se fait la reproduction des petites cornes.

La Nature ne suit pas toujours exactement la même marche, dans la régénération des cornes de l'escargot. Il arrive sourent que le tronçon, au lieu de s'arrondir, devient aigu, et s'allonge insensiblement. De l'extrémité sort un point noirâtre, qui est l'œil. Au bout de quelcque tems, cette extrémité s'ulargit, et forme 


\section{氵oo LA COCHLIOPÉRIE.}

une espèce de petite boule, qui porte l'œi à sa surface antérieure. Du reste, tont s'exécute de la manière que je viens de dire.

Le nombre des parties qui composent la portion rejpoduite, diffère-t-il, ou ne diffère-t-il point, du nombre de celles qui composaient la partie retranchée? La dissection la plus scrupuleuse n'a pu m’y faire découvrir la moindre différence.

Quel que soit le nombre des cornes enlevées, quel que soit l'endroit de la corne où l'on fait la section, la reproduction a toujours lieu. Mais, comme la Nature s'éloigne ici des règles qu'elle observe sur les autres animaux, dans des régénérations de même genre, elle ne répond pas toujours à l'attente de l'observaleur.

Au lieu de couper les cornes seules, sij l'on abat toute la tête d'un escargot. 


\section{LA COCHLIOPÉRIE. 101}

on en roit sortir une nouvelle. Cette reproduction s'opère d'une façon tout-à-fait singulière. Par exemple, quand on coupe la tête et la queue à un vèr de terre, les parties reproduites forment un tout organicque et entier, c'est-à-dire, une espèce de tête et de queue parfaitement semblables aux portions retranchées, si ce n'est qu'il manque à leurs anneaux un développement plus complet. Du tronc de l'escargot, il ne sort pas de même un tout organique, qui embrasse en entier les diverses parties qui composaient la première tête. Ces parties commencent souvent par être séparées l'une de l'autre; ou bien elles sont reproduites l'une après l'autre, à différentes époques; et re n'est qu'après un espace de tems plus ou moins long, qu'elles s'unissent, se consolident, et forment un tout assez ressemblant iे leur premier modele. Les observations suivantes feront mieur comprendre ce que je veux dire. 


\section{LA COCHLIOPÉRIE.}

La reproduction de la tête n'est quelquefois qu'un petit globe, qui contient les élémens des deux lèvres, des deux petites cornes, de la bouche, et des dents. Ce petit globe, situé au centre de la section du tronc, ne contient pas les autres parties, telles que les deux grandes cornes, et la portion antérieure du pied (ou de l'empatement), laquelle est conıiguẻ à la tête des escargots qui n'ont point été décapités.

Dans d'autres individus, on voit d'abord le tronc reproduire la grande corne droite, jusqu'à la longueur d'une ligne, avec un œil à son extrémité. Par-dessous, dans un endroit séparé, on aperçoit ensuite les premiers indices des lèvres.

Dans d'autres encore, la reproduction se manileste par un nœud de trois cornes, dont deux ont déjà leur grosseur et leur longueur naturelle, tandis que Is troisième se montre à peine. 


\section{IA COCHIIOPÉRIE. IO3}

Enfin tanlôt ils ne reproduisent d'abord qu'un petit bouton, qui renferme simplement les lèvres naissantes; tantôt ils montrent tout d'un coup la tête entière, à la réserve d'une ou de plusieurs cornes; et tantôt ils reproduisent premièrement les deur grandes cornes, ou bien les deux petites, ou une grande et une petite.

Il y a aussi des escargots qui restent toujours avec le tronc seul, sans aucune marque de reproduction, quoique leur section ait été faite en même tems que d'autres, dont les différentes régénérations ont eu lieu.

J'ai vu, au contraire, dans quelques escargots, que la partie reproduite ressemblait abolument à la partie retranchée, à l'exception d'une ligne grisâtre perpendiculaire à l'axe du cou, laquelle indiquait précisément l'endroit où les ciseaux avaient passé dans l'opération. Ce n'est pourtant pas toujours une simple 


\section{$10^{\prime}$ LA COCHLIOPERIE.}

ligne qui marque l'endroit de la section. C'est quelcquefois une rainure profonde, d'une couleur blanchâtre. Cette rainure est perpendiculaire à l'axe du cou, si la section a été faite perpendiculairement; elle est oblique, si la section a été oblique. Dans ce dernier cas, il arrive souvent que, du côté où l'on a retranché une plus grande portion de la tête, la rainure est plus profonde, et même trèscreuse, tandis que de l'autre elle ne conserve aucune marque de l'opération, si ce n'est la ligne grisâtre dont je viens de parler.

Quoique le tems efface pen-à-peu ces rainures, la marque de la section, c'està-dire, cette ligne grise ou creuse, parait sur le cou des escargots, pendant plus de deux ans. On voit même quelquesuns d'enir'eux à qui ce long intervalle ne sulfit pas, pour la reproduction totale de la tête: il y manque souvent une corne 


\section{A COCIILIOPÉRIE. IOS}

ou plusieurs. Quelquefois ces têtes reproduites n'ont pas leur grosseur naturelle, ou bien elles sont monstrueuses et remplies de tumeurs. J'ai encore souvent observé aux parties reproduites, une certaine difformité, qui consiste dans une disposition de quelques organes de la tête, qui n'est point naturelle (5).

D'après ce que je viens d'exposer, on se formera aisément une idée des différentes bizarreries qui peurent avoir lieu dans la reproduction des têtes d'es-

(5) Les tumeurs et les monstruosités donk parle Spallanzani, prorenaient surement de ce que ses escargoḷ élaient exposés à la pluie, ct de ce qu'jl y en a eu qui l'ont reçue trop souvent dans leur coquille. J'ai essayé de noursir des escargots mulilés, en versant, dans leur coquille, le suc vif de quelquies autres escargols; ils out pompé cet aliment par l'anus, el m'ont fait roir quelque tenis après, les formes hideuses dont parle le Naturaliste italicn. 


\section{LA COCHLIOPÉRIE.}

cargots, quoique toutes tranchées dans un même tems. Mais sont-ce de réritables bizarreries, c'est-à-dire, des erreurs, des jeux de la Nature? Ou ne sont-ce que des changemens fondés sur des lois constantes et invariables? Croirons-nous que c'est de la section plus ou moins grande, perpendiculaire ou oblique, que résulte la loi qui préside à la future reproduction? Quelqu'idée qu'on en ait, je pense cquau moyen d'un examen exact et scrupuleux, on pourrait arriver à découvrir le systême de la Nature, et faire entièrement disparaître toute espèce de bizarrerie apparente, dans ce genre de reproductions.

Lorsque je me fus aperçu que les têtes régénérées mange ıient, je me persuadai facilement que la reproduction de toutes les parties qui les composaient, était parfaite. Pour m'en assurer, j'eus recours à la dissection. J'ai remarqué que, dans 


\section{LA COCHLIOPÉRIE. $10 \%$}

les escargots, qui, par leur figure extérieure, annoncent avoir reproduit toute la partie retranchée, non-seulement la nouvelle tête posiède les organes similaires et dissimilaires dont j'ai parlé plus haut, mais encore plusieurs autres dont je n'ai point fait mention, lesquels réunis aux premiers, forment une tête entière. Chaque partie de la nouvelle régénération se joint si bien aux parties anciennes, qu'à regarder attentivement l'escargot, on ne croirait pas cqu'il eût été mutilé, si la ligne grisâtre, qui traverse son cou, n'était un indice assuré de la section.

Ces choses se remarquent toujours , soit qu'on fasse la section au-dessus du cerreau, soit qu'on la fasse au-dessous, dans la moëlle spinale. Lorsqu'on la fait en ce dernier endroit, on voit la reproduction de la partie retranchée de la moëlle, et celle d'un nouveau cerveau, 
108 IA COCHLIOPÉRIE.

d'où sortent les dix nerfs que j'ai indiqués.

Après avoir su que l'escargot reproduit sa tête, il était naturel de conjecturer qu'il reproduirait aussi les autres parties de son corps, dont la structure est moins composée. Tels sont ce collier émi. nent qui orne, et environne le dos de l'escargot, lorsqu'il sort de sa coquille; et ce pied plain et large, sur lequel il porte son corps en marchant. En effet, si l'on mutile ces deux parties, elles se régénèrent parfaitement.

Ia faculté de reproduire les membres retranchés, est-elle accordée à toutes les surtes de colimaçons terrestres? Oui. J'ai vu du moins celle reproduction, dans toutes les espèces d'escargols que j'ai pu examiner. Il y en a même une, dont je parlerai ailleurs, que la Nature a privilégiće d'une manière particulière. Ies limaçons sans corquille, dont Rédi 


\section{LA COCHLIOPERIE. 109}

a décrit si agréablement les amours, mériteraient une place dans mes recherches. Mais l'attention que j'ai donnée aux premiers, m’a laissé peu de loisir pour vaquer à l'examen de ceux-ci. Le résultat du petit nombre d'expériences faites sur la limace est qu'elle reproduit ses cornes arec la même facilité que le colimaçon. La reproduction des autres parties de la tète est beaucoup plus difficile dans les limaçons sans coqquille. que dans les escargots. 



\section{LA COCHLIOPÉRIE.}

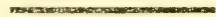

SECONDE PARTIE,

S U R

LN SPÉCICIQUE ANTI-HERNIATRE.

\section{ARTICLE PREMIER,}

\section{$D E I A$ POURPR.}

Tands que je m'occupais à faire des expériences sur les escargots, pour éten1re et vérifier les découvertes des reproductions animales, je me rappelai que les Ancieris tiraient d'une espèce de colimaçons, la teinture de leur belle pourpre, ainsi que je l'ai dit dans la première partie de cet ouvrage $\left(^{*}\right)$.

Les Anciens avaient une si grande ad-

(*) Premièro Parlic, arlicle V. 


\section{LA COCHLIOPERTE.}

miration pour cetle teinture, que ce n'est point exagérer de dire, qu'ils la portaient jusqu’à l'evtravagrance. Horace los en raille lui-même dans une de ses Epîtres:

\section{......... Quibus oblitus actor}

Quium stetit in scena, concurrit dertera lave. Dixit adhue aliquid? Nil sanè. Quid placet ergò?

Lana Tarentino violas imilata veneno.

" Dès qưun acteur richement vêtu entre " sur la scène, on se récrie, et l'on frappe "des mains. - Hé ! qu'a-t-il dit? - Pas " 1 m not. - Qu'admirez-vous donc?" L'acteur qui paraît, porte une robe "violette teinte dans la pourpre de Ta"rente $\left(^{*}\right) \cdot$ "

Il semblerait, par ce passage du poëte latin, que la pourpre de Tarente, ville d'Italie, était supérieure à toutes les autres pourpres. Néanmoins il est cons-

(^) Epitres d'Horace, livre II, Epître 1. 


\section{LA COCHLIOPÉRIE. $\quad 113$}

tant que la plus belle des étoffes de ce gente, était celle que l'on fabriquait dans la ville de Tyr, en Phénicie. Pline l'ancien, parlant des diverses sortes de pourpres, dans son Histoire Naturelle, place au premier rang la fameuse pourpre de Tyr, et dit que c'était la meilleure de toute l'Asie. Il ajoute que la meilleure pourpre d'Afrique, était celle de $\mathrm{Me}$ ninx ou Meninge, île de la Méditerranée, avec celle de la côte de Gétulie, sur l'Océan; et que la meilleure pourpre d'Europe était celle de la Laconie, en Grèce $\left(^{*}\right)$. Mais il observe que celle de Tarente fut beaucoup recherchée, pendant quelque tems; et l'époque dont il parle tombe précisément à celle ou florissait Horace $\left.{ }^{* *}\right)$.

(*) Hisioire Naturelle de Pline, livre IX, shapitre 56.

(*) Ibidem, live IX, chapitre 59. 


\section{I14 LA COCHLTOPÉRIE.}

On trouvera, daus l'Histoire du Commerce, par Rollin, à l'article de la Pourpre, le nom de presque tous les anciens autcurs qui cn ont parlé, aussi bien que de l'espèce de limaçons qui la produisait $\left({ }^{*}\right)$. On peut roir aussi dans l'Oricrine des Lois, des Arts et des Scicnces, par Gognet, ce que dit cet historien sur les couleurs employees à la teinture des étoffes $\left(^{* *}\right)$.

Raynal, qui a traité celle matière, et que je lisais, lorsque je faisais mes premières expériences, est l'auteur qui a fixé mon altention sur le suc ou la liqueur des hélices terrestres, en général.

${ }^{\star}$ ) Histoire ancienne de Rollin, tome $X$, article 8 , la Pourpre.

${ }^{* *}$ Origine des Lois, des Arts et des Sciences, par Goguet, tome II, livre II, section I, chapitre 2, arlicle 1, des Couleurs employés à la teintute des étoffes. 


\section{A COCHLIOPERIE. I15}

Voici ce qu'il dit de la pourpre, dans son Histoire philosophique et politique des ćtablissemens et ducommercédes L'uropéens dans les deux Indes:

"C'est une particularité aujourd'hui " conuue, que, sur la côte de Guaya" quil, aussi bien que sur celle de Gua"timala, se trourent les limaçons qui " clonnent cette pourpre si célébrée par "les Anciens, et que les modernes ont " cru perdue. La coquille qui les ren" ferme est attachée à des rocher's que "L la mèr baigne. Elle a le volume d'une "grosse noix. On peut extraire la liqueur " de cet animal de deux manières.

"Les uns le tuent, après l'avoir tiré "de sa coquille, le pressent ensuite arec " un conteau, depuis la tête jusqu'à la "queue, séparent du corps la partie oì " s'est amassée la liqueur, et jettent le " reste. Quand cette manœurre, répétée " sur plusieurs limaçons, a donné une 


\section{LA COCIILIOPEPIE.}

"certaine quantité de liqueur, on y "plonge le fil qu'on veut teindre, et " l'opération est faite. La couleur, d'a" bord blane de lait, devient verte, et "n'est pourpre que lorsque le fil est sec. "Ceux qui n'aiment pas celte mé" thode, tirent en partic l'animal de sa " coquillc, et lui font rendre sa liqueur " en le comprimant. On répète cette " opération juscqu'i quatre fois, en dif"ferens tems, mais tonjours moins uti" lement. Si l'on continue, l'animal " meurt, à force de perdre ce qui fai$"$ sait le principe de sa vie, qu'il ne peut " plus renouveler, par épuisement.

"On ne commit point de couleur qui " soit comparnble à celle dont nous par"lons, ni pour l'éclat, ni pour la du"rée. Elle réusit mieux avec le coton, " quavec la laine, le lin ou la soie (*)."

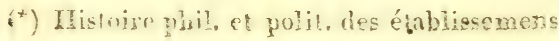




\section{A COCHLIOPERIE. 117}

Ia Harpe fait le même récit, et développe un peu plus la manutention de la pourpre moderne, dans son Histoire générale des Voyages, en traitant de l'histoire naturelle de l'Amérique méridionale $\left(^{*}\right)$. Réanmur a aussi parlé de la découverte d'une nourelle teinture de pourpre, en Angleterre et en France, dans les Mémoires de l'Académie cles Sciences (**). Duhamel, après lui, a entrepris plusieurs expériences sur la pourpre, dont il est fait mention dans le même ouvrage $\left(^{* * *}\right)$. Je me borne ì

et du commerce des Européens dans les deux Indes, par Raynal, tome IV, lirre VIII, article 28 .

${ }^{*}$ Histoire générale des Voyages, par Ia Harpe, tome XIII.

(*) Mémoires de l'Académie des Sciences, année 1711 , page 166.

(***) Ibidem, année 1736 , page 49. 


\section{LA COCHLIOPERIE.}

jenvoyer les curieux à ces auteurs. Le texte de Raynal, comme je l'ai dit: ayant été le sujet de mes réllexions, je dois le reporter de préférence et uniquement, afin qu'on apercoire micux l'enchainement de mes idees, avec la raison de mes procédés ou rle mes moyens. 


\section{LA COCHLIOPÉRIE. 119}

\section{A R T I L E II.}

RECHERCHES SUR LE SUC DES ESUARGOTS DE VIIGNE.

J'AI d'abord fait quelques essais de teinture, avec du suc d'escargots de vigne. J'ai remarqué que celte liqueur s'attache si fortement au linge, qu'il est extrêmement difficile d'en enlever la couleur bleuâtre, soit par l'eau bouillante, soit par le saronnage, soit par l'urine, soit par la lessive.

La propriété singulière de ce suc m'a lait soupçonner qu'étant appliquée extérieurement, dans certaines infirmités, il pénètrerait facilement la peau, et se répandrait dans toute la partie malade. Considérant ensuite que l'on mange des escargots, et que les personnes qui ont la poitrine faible en boivent le jus, quel. 


\section{LA COCHLIOPERIE.}

quefois même le suc pur; je n'ai poini balancé à croire que ce suc, insinué dans les muscles, ne devait pas être plus nuisible que la chair cuite, ni que le bouillon et la liqueur naturelle de ces animaur, introduits dans l'estomac. Il me restait à découvrir l'espèce d'infirmilé que cette liqueur pouvait guérir. Sa viscosité, sa force astringente ou de contraction, et ses facultés reproductives, me firent aussitôt présumer qu'il fermerait, de quelque manière que ce soit, les ouvertures herniaires. Cette pensée me parut d'autant plus digne d'attention, qu'il n'y a point de spécifique connu contre cette maladie, et que la moitié du genre humain en est affligée. Elle devint l'objet de mes rèflexions dans des promenades solitaires; et je ne cessai de m'en occuper, ¡usqu'à ce que j'en eusse suffisamment examiné les développemens, et approfondi les conséquences. 


\section{LA COCHLIOPÉRIE. I2I}

\section{A RTICLE III.}

ESSAIS SUR LA GUÉRISON $D E S \quad H E R T I E S$.

Lorsque j'eus bien médité les procédés dont je devais faire usage, pour cette espèce de cure, j'entrepris de traiter deux personnes hernieuses, ou incommodées de descentes, et j'eus le bonheur deles guérir radicalement, dans l'espace d'environ trois mois. L'une de ces personnes, âgée de vingt-quatre ans, avait une hernie de la grosseur d'une areline, ou, plus exactement, d'une demi-noix. L'autre, âgée de trente-six ans, avait une hernie qui élait une fois plus considérable.

Quilque tems après, j'écrivis à un célebre chirurgien de Paris, pour lui faire part de ma découverte. Je l'abusai, 


\section{L A COCHLTOPERTE.}

comme on doit le croire, sur le prix ei la nature du remède ; et je lui demandai s'il voudrait se charger de l'administrer, moyeunant une remise homnêle que je lui ferais, sur les recettes. Ce chirurgien, qui traitait particulièrement les hernies, me fit réponse, qu’il désirait connaître mon remède, avant de prendre aucun en gagement avec moi; et qu'il ne se chargerait des cures que je lui proposais de faire, qu'aulant que je consentirais à promulguer mon secret, au bout de deux amnées, ce terme, disait-il, suffisant pour faire ma fortume. Ces deux propositions me parurent un refus blâmable, couvert du voile de l'amour du bien puiblic. J'y fis la réplique ambiguë qu’elles devaient aroir, et il n'en fut plus question.

Cependant je tâchai de decouvrir secrètement d'autres malades, pour m'assurer de l'efficacité de mon remè le. On no'n procura quatre, de différens âges . 


\section{I.A COCHLIOPÉRIE. 123}

dont trois suivirent mon traitement, le quatrième ayant quilté, par ordre du Gourcrnement, le pays cque j'habitais. De ces trois malades, l'un a été guéri en deux mois; les deux autres, en trois on quatre mois.

D'ilprès le succès de ces nouvelles, épreuves, rien ne sopposail plus à ce crue je n'annonçasse comme possesseur d'tu spécificue anti-herniaire; mais je n’ai jamais pu me résoudre à changer le cours de mes occupations. Chacun a ses gouts, ses inclinations, son genre d'existence, et je sens que je serais peu propre à traiter cette espèce de maladie, quelque brillante fortune cu'une pareille profession dût m’assurer. Ma décourerte néanmoins m'a paru trop ulile à la nature souffrante,pour la garder en moi-même, jusqu'à ce que je rencontrasse un chirurgien bénérole, digne de ma confiance. J'ai même cru que, retiré du monde comme 


\section{I24 IA COCHLIOPERIE.}

je le suis, par habitude et par principes, je devais penser au moyen de ne pas laisser un secret aussi salutaire, trop long-tems exposé aux hasards incalculables de la rie. C'est pourquoi, son rapport au systême général de mes expériences m'ayant permis de le joindre à ce Recueil, je n'ai point balancé à saisir cetle occasion de le publier, et je rais su faire lommage à l'luumanité. 


\section{LA COCHLIOPÉRIE. I25}

\section{A R T ICLE I V.}

\section{TRAITEMENT DES HERNIES.}

Le malade, avant d'entreprendre sa gुuérison, doit comnaître de quelle espèce de hernie il est affligé, sur-tout quand elle est fort ancienne. Il doit consulter, à ce sujet, un chirurgien ou un homme de l'art. Si la hernie rentre facilement, et qu'il n'y ait point de danger à la contenir dans le bas-rentre, le malade pourra se traiter lui seul, sans aucune autre consultation. Si la hernie ne rentre pas facilement, il faut que le chirurgien la fasse rentrer avec les précautions conrenables, en y appliquant l'emollient d'usage, qui est un cataplasme de mie de pain et de lait, ou tout autre; après quoi le malarde pourra encore se traiter lui seul. Si la hernie, quelle qu'elle soit, ne peut 


\section{LA COCIILIOPÉRIE。}

rentrer par aucun moyen, ou s'il est dangereux de la renfermer dans le corps; le nualade ne fera point usage du spécifique dont je vais parler, il pourrait augmenter son mal. Ce n'est point ici le remède qui se trouve en défaut, c'est la chirurgie qui ne peut faire rentrer les intestins, ou les dégager de lemrs humeurs putrides et malfaisantes. Au reste, cet inconvénient n'aura jamais lieu par la suite, puisqu'une hernie n'aura jamais le tems de vieillir, de s'accroître, et de s'envenimer.

Lorsqu'un malade, de l'un ou de l'autre sexe, aura pris les informations que je viens de prescrire, il se fera faire un bandage à coquille, qui enveloppera loute la circonférence de sa hernie; c'estì-dire, que la pomme du bandage, au lieu d'être arrondie ou en bosse, sera un peu concave, pour recevoir une espèce de pelit gode!, d’un diamètre égal à celui 


\section{LA COCHLIOPÉRIE. I27}

de la hernie. Peut-être est-il indifférent que ce godet soit d'une matière dure quelconque, telle que la porcelaine, la faience, le verre, le fer-blanc, le bois de poirier, le buis, etc.. Il semble pourtant préférable qu'il soit de porcelaine, de faience ou de verre, afin que la liqueur qu'il contiendra ne puisse pas le pénétrer, ni perdre une parlie de sa vertu, ni être altéré en aucume façon. La profondeur de ce godet sera d'une ou deur lignes, et de trois lignes pour les plus fortes hernies. Sa concavité aura la forme d'une calolle sphérique, ou d'un bassin de balance. Ses bords, un peu évasés, afin de ne point incommoder le malade, prendront bien la rondeur de la pomme du bandage, sur laquelle ils ne formeront aucune inégalité, lorsque le godet scra placé dans la coquille. On conçoit que ce godet devra ressembler à un petit chapeau pastoral. Je fais connaître cette forme ou 


\section{$\$ 28$ LA COCHLIOPERIE.}

cette disposition, comme étant la plus avantageuse. Néanmoins je me suis toujours servi, à deiaut de pareils vases, des godets de faience qu'on emploie ordinairement pour dessiner er laver des plans.

Quelle que soit l'espece de godet dont on fera usage, on le remplira de laine, de coton ou d'étoupe, que l'on changera tous les deus jours. Autant que une le fait commaitre le texte de Raynal sur la tcinture de powpre, il me semble gne la laine raut miens que le coton, puisque la liqueur la penètre moins, et qu'il s'agit senlement de contenir celte liqueur dans le godet. Je crois meme qu'elle vaut micux que le lin on le chanvre, parce que sa nature grasse doit la rendre moims pénéralıl: que res végétaux. Il serait facile d'en faire l'expérience. An surplus, je préviens encore que jai toujours employé de la laine, parce que 


\section{LA COCHLIOPERIE. I29}

sa vertu médicinale est d'ailleurs commue.

On se procurera environ deux cents escargots de rigne; c'est le nombre çu'il faut généralement, pour guérir une hernie ordinaire (6). On les nettra dans un lieu frais, ou ils puissent prendre de la nourriture, parce qu'on n'en sacrifie que deur ou trois par jour. Si on le préfere, on se les procurera par douzaine; on les cnfermera dans une toíte, ou dans une cage de bois; et l'on mettra cette cage sur une fenêtre, ou dans une cave, un cellier, un rez-de-chaussée, une cour, un jardin, un buisson. Les escargots de vigne se trouvent dans les rignes, en grande abondance; il y en a aussi dans

(6) Tous les escargols serainl également bons à cmployer, particulierement les escargots de jardin; mais il en fandrait un nombre trois ou quatre fois plus considérable, el peut-ètre davantage. 


\section{\$3o LA COCHLTOPERIE.}

les haies, el dans certains bois. Les her boristes ei les apolhic ires en rendent; il en manque rarement dans les manhés publics des villes. Ceux de ces animaur qui ne remplissent pas leur cosnilie, et qui sont légers, ne conviennent pas pour le traitement (f'i nous occilpe; ils sont trop maigre; , trop desséchés. Lorsqu'ils ne coûtent rien, cela est asicz indifférent, parce qu'on en immole six ou huit par jour, au lieu de deux ou trois. Mais, si on les achète, on cloit $y$ laire attention, ces sortes d'escargots se rendant plus ou moins chèr, en détail, selon les années et les saisons.

Le prin!ems, qui est l'époque où les escargots remplissent le mieux leur coquille, est aussi l ı saison que l'on doit preférer pour traiter les hernies. D'abord à cause de la facilité qu'on a de se procurer ces animaux; ensuite à cause des chalıurs, qui dilatent les pores des 


\section{LA COCHLIOPERTE. IZI}

tégumens commıns; enfin parce crue c'est la température la plus propre aux régénératicns. Toutes les guérizons dont j’ai jarlé ci-dessus, ont été commences avec le printems. Elles eussent été vraisemblablement plus lentes, si le traitement avait eu lieu en automme, ou en hirèr. Elles eussent çé aussi un peu plus dispendieuses. Quand on roudra y meltre la plus srande économie, on pourra se servir deux ou trois fois du même escargot, parce qu'il ne meur't pas des blessures quoon lui fait. Il suffira de marquer sa coquille, pour ne pas le cono fondre avec ceux dont on naura point encore fail usage, et de le jeter sur l'herbe, ou dans un liuisson, afin cqu'il se rétablisse par de nouveaux alimens.

Tous les jours, le malade, avant de se lever, et après s'ctre couché, retirera le godet de dessuus son bandage, prendra un escargot, et le blessera en divers 


\section{I32 LA COCHLIOPÉRIE.}

sens, par intervalle, arec une cheville d'un bois dur, qui aura la forme d'un poinçon. A chaque blessure que recevra l'escargot, par l'ouverture de sa coquille, il jetera une eau, tantô! bleuâtre, tantôt gris de perle, qu'on recevra sur la laine du godet.

Cette eau est le sang du colimaçon. En effet, si l'on brise la coquille d'un escargot, pour le meitre à nu, ou si l'on Lire cet animal à moitié hors de sa reiraite, en le perçant avec un poinçon, et qu'on l'ouvre sur le dos, un peu audessus de la jonction du corps et de la spire ou queue; son cocur, facile à recomaitre, par. son moniement de diastole et de sistole, sera d'une coulcur semblable à l'cuu qu'on fait jeter au testacce, par incision. Rigoureusement parlant, ce viscere est rougeâtre, el d'unc forme approchante de celle du cocur de tous les animaux. Mais il est adhéreni 


\section{IA COCHLIOPÉRIE. I33}

à un petit globule lenticulaire, un peu conique, de la même couleur que son sang, et dont le mouvement péristaltique est ordinairement beaucoup plus sensible que celui de la masse charnue à laquelle il est joint. Ce petit globule se nomme l'oreillette du cour. J'en ai observé le mouvement, pendant plus de cinq mimutes, après aroir séparé le cœur dı reste des autres parties organiquues de l'animal.

Pour ne pas trop m'écarter de mon plan, je n'en dirai pas darantage à ce sujet. On trouvera de plus grandes inssructions, sur le sang, le cœur, et l'anatomie en général des escargots, dans le beau Mémoire sur la Limace et le Colimacon, par George Cuvier, secrétaire perpétuel de l'Institut, tome VII des Annales du Muséum d'histoire naturelle, cahier 38.

Lorsque les coups de poinçon que reI 2 


\section{LA COCHLIOPÉRIE.}

cevra un escargot, ne lui feront plus rendre qu'une écume épaisse, on cessera de le percer. Ce serait en vain qu'on le ferait souffrir davantage, pour en obtenir du sang, la seule liqueur de ce testacée qui ait la propriété que j'annonce. S’il n'en domne point assez, il faudra en blesser un second, chose à laquelle on sera trèsrarement obligé. Il n’y a que les jours où l'on changera de laine, qu'il sera absolument nécessaire d'employer deux beaux escargots, ou trois de moyenne grrosseur. Ainsi, en supposant que la hernie fut trois mois complets à se guérir, il est évident qu'il ne faudrait que deux cent ving-cinq gros escargots, pour 1out le traitement, sans jamais se servir des mêmes.

Comme il peut arriver quelquefois qu'on n'ait point de laine, pour fixer la liqueur dans le godet, il est bon de savoir die quelle manière on devra y suppléer. 


\section{LA COCHIIOPERTE, I35}

La liqueur de l'escargot étant reçue dans une soucoupe, ou un autre vase semblable, on l'y battra vivement, avec une spatule de hois, et elle se convertira en une espece d'onguent, qui prendra la forme d'une boule. On étendra cette boule dans le godet, on applicpuera le gociet sur la hernie, et l'on serrera le bandage un peu plus qu’à l’ordinaire, parce que l'onguent se remet assez promptement en eau. Cet expédient ne doit pas engager à négliger la laine. Non-seulement elle procure une chaleur nécessaire à la partie malade, mais elle dispense de trop serrer le bandage. Je le fais bien remarquer: ce que je viens de dire, est pour le seul cas où l'on n'aurait pas delaine, au moment qu'il faudrait en changer.

Quant à ce renouvellement, le malade ne doit y apporter aucune négligence. Avec le tems, la liqueur fermente dans 


\section{IA COCHLTOPEIIE.}

le godet, et pour peuqu'elles'y corrompe, elle répand une odeur infocte vrainent insupportable. Il serait sans doute trèsdangereux de faire entrer ce fluide empesté dans le bas-rentre. Je m'exprime en termes peu ménagés, afin qu’on y prète plus d'attention, et qu'on ne me rende pas responsable des fautes d'une parcimonie arengle. Je ne prescris pourtant pas de changer de laine tous les matins; cela ne m’a point paru néces aaire, mêne dans les pilus forles chaleurs de l'été.

Chaque fois que l'on versera de nouvelle liqueur dans le godet, on le fera sans déboucler le bandayg. On se contenıera de le soulever un peu, pour retirer le vase, et l'on posera la pomme du bandage à côté de la hernie, pour ne pas le tacher. Il va sans dire, qu'on devra être toujours couché sur les reins, dans cet instant; et que s'il prenait au malade une envie de tousser ou d'éteruuer, il ne pour- 


\section{A COCHLIOPERIE. I37}

rait meltre trop promptement son poing fermé sur la hernie, pour empêclier les intestins de sortir, et de rouvrir sa blessure.

Le godet étant suffisamment rempli de liqueur, on le posera sur la partie malade, toujours bien exactement à la même place. Ensuite, pour plus de propreté, on le couvrira d'un linge blanc, et l'on y remetira la coquille du bandage. Ce bandage, sans être trop serré, derra l'être assez, pour empêcher le fluide de couler entre la peau et le vase, quelque posture que l'on prenne dans le lit, ou dans les différens exercices du jour. J’ai prévenu que chaque goutte de la liqueur anti-herniaire produit, sur le linge, une tache presqu'ineffaçable. Comme il est inutile de se mettre dans le cas de divulguer que l'on se traite d'une hernie, on fera bien, pour éviter toute espèce d'explication à ce sujet, le purter, jour et 


\section{IA COCHLIOPÉRTE.}

nuit, un caleçon, entre la peau et la chicmise.

Le malade ne sera exposé à aucune sujétion, à aucun régime quelconque, dans tout le cours de son traitement. Voici les seules précautions qu'il devra prendre. Il se rasera la partie malade, tous les quatre jours, en motillant le poil avec la liqueur du godet dont il changera la laine. Il ne laissera jamais la hernie long-tems décourerte, dans ses pansemens, afin d'eviter le refroidissement de la peau. Il appuiera plus ou moins sa main sur son bandage, toutes les fois que, le jour ou la nuit, il toussera, éternuera, ou fera un effort quelconque. S'il arrive que les. bords du godet entament la peau, ce qui proviendra de ce que le malade aura lais sé trop croître le poil, ou de ce que le godet sera mal fait; il faudra suspendre le traitement, jusçu’à ce que l'écorchure soit guérie. 


\section{A COCHLIOPÉRIE. I3Y}

Cetle inteription durera deux nuits $e_{t}$ un jour, ou tout au plus trois nuits et deux jours de suite, selon les cas. Alors, pendant la nuit, le malade ôtera entièrement le bandage, s'il peut le faire sans que les intestins passent au travers de l’auneau, ce qui dépendra de la position qu'il aura coutume de prendre dans son lit. Pendant le jour, il portera le bandage à sec, en remplissant toute la coquille d'un tampon de laine, et meltant un petit linge sur la hernie. Remarquez que je place la nuit avant le jour, dans chaque interruption, afin d'aroir toujours l'avantage du nombre des nuits sur celui des jours. 


\title{
4 LO LA COCHLIOPÉRIE.
}

\section{A R T I L E V.}

\author{
$R \dot{E} S U L T A T$.
}

PAR cette espèce de traitement, une hernie ordinaire doit être guérie en trois mois, ou tout au plus en quatre mois, à moins que le malade n'ait quelque vice qui s'oppose à la fermeture de l'anneau.

On s'apercevra que la guérison est complette, lorsque, mettant le doigt sur l'anneau, on le trouvera fermé, ou pres‘qu'entièrement fermé. J'ai abandonné le traitement d'un jeune homme de vingt-buit ans, lorsque je pouvais encore passer le petit doigt dans l'anneau; la nature seule a achevé la guérison. Le premier jour que jai vu sa blessure, la circonférence de l'ammeau était assez grande pour que j'y fisse entrer trois doigts. 


\section{LA COCHLIOPERIE. IA}

\section{A R T I C L E V I。}

\section{INSTRUCTION}

$S U R I A C O N V A L E S C E N C E$.

Qcorq̨ue l'anneau soit fermé, autant qu'il doit l'être, il est bon que le malade continue de porter son bandige, pendant six semaines ou deux mois. Il en remplira la coquille arec de la laine, et recouvrira cette laine avec de la peau, comme cela se pratique ordinairement. Plusieurs raisons exigent qu'il ne quitte pas tout d'un coup cette espèce de ceiniure. Une persomne qui est guérie d'une pareille infirmité, croit, de tems à autre, que sa hernie va reparaitre. Ce jeu de la nature n'inspirera aucune crainte, quand. on portera un bandage. Il n'en serait pas de même, si la partie malade était enfièrement libre. D'ailleurs il est indiso 


\section{I/2 LA COCHLIOPËRIE.}

pensable de laisser raferu.ir la plaie, dans l'ètat où elle se troure actuellement, avant de fermettre aux miscles qui l'enviromuent, de prendre tout le jen qui leur est naiurel. Un effort impréru pourrait produire plus de déât qu'il n'y en avait mparavant, si l'on aisandonnait trop tôt à lui-même l'anneau hunecté de la liqueur anti-herniaire.

Lorsque le malade sera assez rétabli pour quitter tout-ì-fail le beuráase, et qu'il l'aura effectirement quite, il ne devra jamais cesser d'appliquer sa main sur sa blessure, quand il fera quelque effort, durant les deux premières années qui suiviont son traitement. Je ne Jui prescrirais pas cette précaution, qu’il la prendrait de lui-même, parce qu'il lui arrivera cinq ou six fois, dans le cours des deux premières années, d'éprouver intérieurement, soit à l'anneau, soit au péritoine, une douleur lente, qui 


\section{A COCHLIOPÉRIE. 143}

Ini ferait croire qu'il est mal guéri, s'il n’était prévenu du contraire. La durée de cette douleur est d'un ou denx jours, quelquefois d'une demi-journée seulement. Je presume qu'elle a pour cause un changement de température, qui influe sur la liqueur renfermée dans les muscles du bas-ventre, comme sur les escargots eux-mêmes, lorsqu'ils se mettent en mouvement. C'est du moirs ce que me fait penser une influence du tems trèsétomnante, sur la liqueur des limaçons quidoment la couleur pourpre. La Harpe dit que les matières qui en sont teintes, pèsent plus à certaines heures du jour qu'à d'autres. Il se pourrait aussi que ces douleurs fussent les suites de la nualadie morale dont j'ai parlé plus haut, puisque tous les sujets que j’ai guéris ne les ont point éprouvées.

Quoi qu'il en soit, si le traitement essentiel d'une hernie, est limité à envirou 


\section{I4 LA COCHLIOPERIE.}

trois mois, je ne crois pas que ces petits accidens, sur tel ou tel individu, puissent autoriser à dire que la guérison radicale d'une hernie dure une ou deux années. Celte guérison est assurée, elle est complette, au moment qu'il n'est plus nécessaire de continuer l'usage du spécifique. Mais la Nalure, toujours lente dans ce qu'clle fait de bien', ne peut oublier ses lois dins une opération de cette importance.

Loin de s'étonner qu'une plaie hero niaire, fermee d'abord par contrainte, fasse éprouver quelquefois, à certaines personnes, l'espece de mal-aise dont il vient d'étre question, il fant que l'on sache, pour prévenir des craintes pu= sillanimes, qu'il est physiquement démontré que tous les convalescens doivent éprouver ces petites souffrances passagères, sans aucune exception. Elles penvent se faire sentir, à différentes époques, 


\section{LA COCHLIOPERIE. 145}

depuis le moment où l'on quilte le bandage, jusqu'à celui où les muscles se trouvent tout-à-fait dégagés du remède, par la transpiration insensible. Tant mieux pour l'être privilégié qu'une constitulion plus ou moins robuste préservera constamment de cet état de gène. Mais, dans un raisomement oir je n'ai point de donnée, je ne reux ni abuser, ni flatter persomne. Quel que soit l'aspect sous lequel ces observations pathologiques se présentent aux yeux de l'ignorance, lhonneur me défend de les omettre, et mes sentimens me disent gu'un homnête homme doit préférer sa propre estime à tout ce qui pourrait lui être le plus avantageux.

Si la cupidité, la malreillance, et d'autres manvaises passions, trourent, dans ce lirre, matière à contester et à embrouiller les taits; ce ne sera donc. plus moi qui aurai tort. Aussi ne me 


\section{LA COCHLTOPRTE.}

déprefirai-je point de la résolution que jai prise de ne repondre à ancune espece de chanenas. Apres aroir dit loul ce qui esl, lout ce qui doil être, lout ce qui peut être, je remets ma cause à un siècle suffismment eclaire sur ce qui concerne les anteurs des belles décourertes. Sans doute quil ne mabandomnera point ì des speculations enmenies. J'ose même espérer cru’il nue fera roir quelques défenseurs, parmi les gens de bien. Celte proteclion, au reste, je la sollicite pius pour le salut commun que pour moimême, quoiquijl fut bien triste que je me urourasse, un jour, dans le cas d'écrire, coume Christuphe-Colomb dans son teslament : C'est ainsi que la reconnaivance du monde paye les services.

\section{F I N.}




\title{
TABLE ANALYTIQUE:
}

\section{DES MATIÈRES,}

\author{
O U
}

IDÉE GEYERALL DE LOLTRAOL.

AVIS. page 2.

Adresse dédicatoire. p. 5.

\section{PREMIERE PARTIE,}

SUR LES REPRODUCTIONS ANIMILES.

\section{A R T ICLE PREMIER.}

DE LA SCIENCE LT DE L'ART. p. 7.

Spallanzani-passe pour étre le premier auteur qui ail dit, que les escargols reproduiscnt leur tète, après qu'on la leur a conpée. p. 7 .

Valmont de Bomare a combattu l'assertion de Spallanzani, par quantiti d'exṕriences. p. ̊.

Voltaire a répété ces expériences, et a laissé la question indécise. p. 10.

Il a coupé la tête à plusieurs escargols et li- 
maces, el il a d'abord cru qu'elle avait élé reproduite en un mois. p. 12.

Il a reconnu ensuite que tontes les téles qui s'étaient régénérées, n'avaient pas été compces arec assez de promplilude. p. 13.

Il a prédil que l'on ferait beacoup d'expériences, après lui, sur ces animaux. p. 14.

Ourrage singulier qu'il a écrit sur les colimaçons. p. 35.

L'Aulem entreprend de vérifier toules les ancirnnes expériencessur les colimaçons. p. 15.

Définition du mot colimaçon. p. 15.

L'Auteur a mutilé, dans ses expériences, environ un millier d'escargots. p. 16.

Ses premieres expériences ont duré dix-huit mois. p. 16.

Réflexions sur ces sontes d'études. p. 16 .

Hórophile a dissíque les corps rivans de roo hommes et femmes. p. 18.

Le Giollo a lié et tué un homme sur une croix, poux le peindre. p. $x 8$.

Parrhasius a fail dćchirer les entrailles d'un vieillard par un vautour, pour peindre un Prométhée. p. 18 . 


\section{DES MATIERES.}

Rondelet a disséqué le cadarre de son propre fils. p. 19 .

Idée général de l'ouvrage. p. 19.

Instrumens dont s'est servi l'Auteur dans ses opérations. p. 20.

\section{A R T ICLE II.}

TÊTE. p. 21.

Manière de couper habilement la tête ì. un escargol. p. 21.

Observation anatomique sur les deux parties internes principales de la tête d'un escargot. 1. 22.

Diverses posilions que peut aroir la cervellé, dans la tète diun escargot. p. 22.

La tête d'un escargot peut vivre mue heure, après avoir été coupée. p. 22.

Ce phénomène comparé à coux qu’on remaxque dans les animaus à sang froid. p. 25.

Harrey, que l'on dit aroir découvert le systême de la circulation da sang, a vu le cœur d'une grenouille conserver son mouvement, pendant une heure, après aroir élé arraché du corps de l'animal. p. 24. 
Remarque sur le réritable auteur de la découverte de la circulation du sang. p. 24, note 1 .

Expóriences de l'Auleur, sur le coeur, lesenIrailles, la tête et le corps d'une grenouille. p. 25 .

L'A uteur a vu le cœur d'ume grenonille conserver son mourement, pendant une henre et rlemie, après aroir élé arraché du corps de l'animal, et avoir été mis au soleil. p. 26.

Celte grenouille a vécu une heure, dans leau, sans coeur ni cntrailles. p. 26.

Une autre grenouille a vécu me heure ot demie, hors de l'eau, après aroir subi la mème opération. p. 27.

Le cocur de celte grenouille a conservé son mourement, pendant plus de quatre heures, dans un lieu frais. p. 27.

Une grenouille décapitée, a vécu vingtquatre heures, dans un pot vide. p. 27.

Question sur le siége de l'âme. p. 28.

Descartes et Péreira ne veulent point que l'homme physique soit assimilé ì la brute, Newton allirme gue le plan de la Niture est 


\section{DES M A TIERS. $\quad 151$}

uniforme, et Salomon dit expressément que les bêtes ont des âmes. p. 28 .

L'Auteur est du mème sentiment que New. ton et Salomon. p. 29.

Réfutation curieuse de tous Jes syslèmes sur le siége de l'âme. p. 29.

Expérieuce effrayanle qui ne laisse aucune xéplique à cette réfutation. p. 5 x.

La philosophie ne peut ni comprendre, ni expliquer les résulials ćlommans de pareilles opérations. p. 31 .

Quel sera fonjours la pensée du rrai philoso phe à ce sujet. p. 52.

Cetle pensće ne confredit point le dogme de l'existence el del'immortalité de l’àme. p. 52.

\section{A RTICLE III.}

CORPs. p. 34.

Le corps d'un escargol peut vivre un an sans léte, et dix-huit mois sans boire ui manger. p. 54 .

Manicre de commaitre si un escargol décapité est en vie, sans le faire sorlir de sa corpuille. p. 55 . 
Un escargot décapité, enfermé dans une vaisse vide, ne reproduit jamais sa tête. p. 36 .

Différentes manières dont on peul faire sortir un escargol de sa coquille, pour s'assurer de cette vérité. p. 56.

Comment on s'aperçoit de la mort d'un escargot, et mème de l'instant où il ra mourir. 1). $3 \%$

Lescargot tient i la colimelle de sa copquille, par une petile membrane. p. 59 .

Procédé par lequel on peut roir cette membrane. p. 3g.

Nulle espèce de recherche, ni d'examen, ne peut faire décourrir une lèle dans un escargot mori, après aroir été décapité, l'eût-il été depuis un an. p. 40.

Un escargot que l'on ne s'empresse pas de jeter aussitôt qu'il est mort, peut infecier tout im appartement. p. 41.

Direrses manières dont son corps se dissont. p. 41.

La chair des escargots se corrompt comme celle des autres animaux, p. 42.

Réflexion sur le roi Darid, à ce sujet, p. \$2. 


\section{DES MATIERES.}

Denx passages de la Bible hébraique, comparés aux mèmes passages de notre Bible, nommée Vulgate. p. 43, note 2.

\section{A R T ICLE I V.}

CoquTLLE. p. 45.

En hivèr, la coquille des escargots est fermée d'un opercule calcaire. p. 45 .

Moyen d'enlever cet opercule, sans blesser l'animal. p. 45 .

Sons cet opercule se trourent plusieurs pellicules transparentes. p. 46.

Lieu où est l'anus des escargots. p. 46 .

Liécritnce it l'encre s'efface difficilement sus la coquille des escargots. p. 46.

Les escargots reconstruisent leur coquille, dans tous les endroits où on la brise. p. 47.

Conjecture sur cette reproduction, dans le cas où l'on meftrait un escargot cnticrement à nu. p. 47 .

La réparation de la coquille, rers l'orifice, est plus lente qu'ailleurs, paree que l'escargot n'y travaille qu'autant que cola preut lui être nécessaire. p. 48. 


\section{A R T I LE V.}

TEMIS LERLUS PROPRE AUX OPERATIONS. 13. 49 .

Le printems est la saison la plus avantageuse anx expériences que l'on peut faire sur les escargots. p. 49.

Dans toute autre saison, les expériences ne domnent généralement que des résultals imparfaits. p. 50 .

Le prinlems est aussi la saison où l'on mange des escargots. p. 50 .

Pays où l'on mange le plus de ces animaux. р. 51 .

Les Grees et les Romains en mangeaient beaucoup. p. 51 .

Pays d'où ils les tiraient. p. 52 .

Manière dont ils les engraissaient. p. 53.

Ils mangeaient aussi les colimaçons qui produisaient l'ancienne pourpre. p. 53.

Leur gourmandise a presqu'entièrement détruit cette espèce de coquillage. p. 53.

Les fricassées d'escargots sont très-célèbres dans l'histoire. $p, 5$ i. 


\section{DES MATIERE. 155}

Une fricassée d'escargols a été cause que Jugurtha, roi de Numidie, a perdu sa couromne. p. 54 .

Le massacre des anciens habitans de l'Amérique a cu pour premier motif, l'usage qu'araient plusieurs de ces perples de manger des escargots et des sauterelles. p. 54.

Les Mahométans d'Alger croient que les escargots sont saints. p. 55 .

Les Juifs, au contraire, croient qu'ils sont immondes. p. 55.

Loi de Moïse, à cc sujet. p. 55.

Le texte hébreu de cette loi, comparé au lexte de la Vulgate. p. 56, note 3.

Dans quelle contrée se trouve le royaume des escargots. p. 57 .

Observation sur les climats qui sont plus ou moins propres aux expéricuces que l'on peut faire sur les escargots. p. 58.

\section{A RTICLE VI.}

NoURRITURL. p. $5 \mathrm{~g}$.

Caisse propre à nourrir des escargots. p. 59 .

Principaux alimens dont se nourrissent les escargols de vignc. p. 60. 
L'escargol mange de la terre. p. 61.

Il mange aussi du papier mouillé et de la páte de farine. p. 62.

Inconvéniens de la caisse aux escargois. p. 62.

Lieu oủ l'on peut melire la caisse en hivèr. p. 63.

Différentes manières de conserver des escargots, dans cette saison. p. 63.

\section{A RTICLE VII.}

EMPATEMENT. p. 64.

Un escargot à qui l'on coupe loute ia partie posléricure de son empatement, la reproduit dans l'espace de six mois. p. 64.

La peau de celle reproduction n'est pas grenue. p. 64.

\section{A R TICLE VIII.}

Cov. p. 65 .

Le cou d'un escargot se recolle, en moins de deux mois, après aroir été fendu transrersalement. p. 65.

Cetle opération a quelquefois une influence singùlière sur les cornes. p. 65 . 


\section{A R T I L E IX.}

FRONT. p. 66.

Le fronl d'un escargot se recolle, en moins de denx mois, après aroir été fendu transi ersale-ment enlre les grandes et les petites cornes. p. 66 .

\section{A RTICLE. X.}

CORNES. p. 67 .

Les cornes d'unescargot se reproduisent, dans l'espace de cinq ou six mois, après avoir été coupées. p. 67 .

Manière de couper les cornes ì un escargot. p. 67 .

Les cornes d'un escargot étant fendues dans inule leur longuenr, se guirissent parfaitement, en deux ou trois mois. p. 68.

Procédé par lequel on réussit à fendre lo: cornes d'un escargot. p. 68.

Question: si les cornes des escargols sont des antennes, des ycux ou des nez? p. 69 .

Le premier av is ne peut manquer d'ètre rrai' mais il ne tire point à consinguence pour lesdons atutres. p. $6 g$. 
Jes cornes des escargots seraient pourtant mieux nommćes des tentacules, ce nom étant spicidement domé an comes des mollusques. p. 6 g.

Ce que c'est que les mollusques. p. 70, note 4 .

Ie second avis semble êlre opposé à l'expérience. p. ro.

Le troisième avis est encore plus opposé à la raison. p. 70 .

Níammoins il est incontestable que les escargols ont un odorat exquis. p. 71 .

Uu sarant moderne a pritendu que le nezdes escargolsestrepandu daus toute leur peau. p. 71 .

L'anatomie ne laisse aperceroir aucun indice de cet organe. p. 71 .

L'Autenr, sans avoir aucun sentiment sur les connes descsrargots, demande si elles ue seraien! pas dies oreilles. p. -2 .

Sur quel principe est fondće cctle question. p. 72 .

Trois mnyens de s'assurer si les cornes des escargots sonl des yeux, des nez, des oreilles, ou si elles sont de siraples antennes, p. 72 .

Pronicre cxpérience relatir eas seus, p. $7^{2}$. 


\section{DES MATIERES. 159}

Seconde expérience relative aux nez. p. 73 .

Troisième expérience relalive anx oreilles. P. 74 .

Si ces trois expériences n'apprennent rien, les cornes sont de simples antenmes, p. 75 .

Romance que les pelits enfans chantent aux escargots, dans la ville de Paris, pour les engager à montrer leurs cornes. p. 75 .

\section{A RTICLE XI.}

CRANE. p. $7^{6}$.

Le crâne, ou tout le sommet de la tèle d'un escargot, se reproduit en sis ou huit mois, après aroir été enlevé d'un seul coup de ciseaux. p. 76 .

Manière de faire cette opération. p. -6 .

MIüler a substitué cette expérience à Ja décapitation totale, et l'a donnée comme un témoignage de l'assertion de Spallanzani. p. 77.

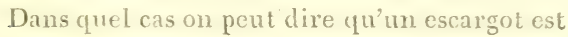
décapilé. [1. 77 .

Cet enlèrement du crâne a fait aperceroix it l' lutrur comment los escarjots aralent leur nomriture, $p, 78$. 
Description de colte fonclion natureth risu es animaux. p. 78 .

Les escargots mangent de la viande cuite el srue, quand on lcur en présente. p. 79.

Quel a élé le but de l'Auteur dans celte dernière épreure. p. 79.

Trail de barbarie exécuté avec un escargot, sur un malheureux rieillard, selon un auteur quimaporle celte ancedote, diapres le président de Thou. p. 79 .

Texte du président de Thon, qui donne l'histoire de ce vieillard dévoré ou rongé par un escarbol, et non par un escargot. p. 80.

Cette fausse citation a conduit l'Auteur à la afeonverte de la reproduction des tétes d'escargots. p. 82 .

\section{A R T I L E X I I.}

REPRODLCTION DE LA TÊTE DES ESCARcoTs. p. $\delta 5$.

Un escargot décapité peut prendre de la nourriture. p. 83.

Liescargol mange quelquefois de la terre et des racines dherbe, sans sorlir de sa coquille. p. 84 , 


\section{DES MATIERES. IfI}

Des escarguts dicapitis, mis dans une caisse vide, y meurent de faim, plutôt que des suites de letir décollation. p. 84.

L'Avteur a u ules commencemens de régŕnérations de tives, à des escargots décapités, qu'il arait mis sux un pré artificiel. p. 84.

Les reproductions animales ue peurent avoir lieu qu'à l'aide des alimens. p. 85.

Mort de tous les escargots mutilés par l'Auteur, lant los dícapités que les autres, vers la fin d'un hivèr. p. 85.

Conjecture sur cet accident. p. 85.

Interuption des expériences de liduleur. p. 86.

U'ne année ensuite, l'Auleur a décapité deux cents escargots, qu'il a jetés dans un bosquct, au commencement du printems. p. 86.

Vers la fin de l'été, de celle mrime année, ces animaux lui ont fail roir des lices reproduites, asse\% ressemblantes it des grains de café, mais les régémalions n'élaient pas complieles. 1. 8\%.

A la fin de l'été, de l'aunée suivanie, les têles ont été entièrement régénérées. p. 87.

Il faut environ deux ans, vil douze mojs de 
belle saison, pour qu'un escargot reprotuise s: tête. p. 87 .

Conclusion sur ce que l'on doit penser de Spallanzani. p. 88 .

Justice rendue à ce naturaliste, malogré les nombreuses expériences qu'on a faites pour l'accuser d'erreur ou d'inhabileté. p. 89.

Riflexion qui pourail suspendre l'hommage que l'Auteur rend à Spallanzani. p. go.

L'Auteur u'a aucun égard à cette objection, parce qu'il pense que, dans le jugement d'un homme, loules les conjectures vagues doiven? céder au sentiment du vrai. p. $9^{1}$.

\section{S U P P L É M E N T}

\section{A LA PREMIERE PARTIE.}

Ohservalions de l'Auteur sur ce Supplémeni. ๒. 95 .

L'escargnt qui ne se nourrit point, ne peut. reproduire sa tête; et celui qui dévore des plantes, n'at pas eu la têle bien conpée. p. gú.

Conjecture sur los succes do Syallanzani. p. 94. 


\section{DES MATIEES. 163}

Procédé par lequel on peut fais commostement la dissection d'un escargot. p. 95.

Des reproductions de la tête et des autres parties, dans l'escargot; et des cornes, dans le limacon sans coquille, qu'on nomme com munément limace; par Spallanzani. p. $9^{5}$.

Analomir de la tète do l'escargyot, selon Spallanzani. p. 95 .

Duscription des yeux de l'escargit, selon cet antens. p. 96 .

Muscles qui font agir sa tête et ses cornes. p. 97 .

Description de sa bouche. p.97.

Manière dout l'escargot reproduil ses cornes. p. 98.

Varialion de la Nalure, dans cette espèce de reproduction. p. ns.

La reproduction a lieu, quel que soit le nombre des cornes, ou la portion des cornes, que l'on coupe. p. 100.

Differenles manizes dont l'escargot repuduit sa têle. p. 100. 


\section{:64. T A B L E}

Quelques escargols décapités par Spallanzani, n'ont jamais reproduit leur têle. p. 105.

Daulres escargois on reproduit une tete parfaitement semblable à la premièrc. p. 105.

Ligne ou rainure qui marque l'endroit ou a été faite l'amputation. p. 103.

Il y a des escargots décapités quil sont plus de deux ans i reproduire compleltement leur tête. p. 104.

A quelyuc-ins d'antreux les reproductions sont monstrueuses. p. 105.

Cause de ces monstruosités. p. 105 , note 5.

Spallanzani n'a vu manger que les têtes régénérées. p. 106.

La dissection lui a prouré que les têles régénéries possedent tous les organes des tites primitives. p. 106.

Le cullicr, et lo pied on empatement de l'es. cargol, se reproduisent aussi, après aroir élé coupés. p. 108.

'Toules les rspeces a'escargots régénìrent les partics de leur corps qui leur sont enlevées. p. 208 .

La limace reproduit ses cormes; mais il est 


\section{DES M A I E P ES. 165}

très-difficile de couper la tête à cet animal, sans quil en ment, an bout de rqualques jours. p. 109 .

\section{SECONDE PARTIE,}

SUR UN SPÉCIFIQUE ANTIMIERVIAIRE.

ARTICLE PRE IIER.

DE LA FOURPRE. p. III.

Raillerie d'Horace, sur l'admiration des anciens pour la pourpre. p. 112.

Distinclion des diverses sortes de pourpres, et quelle était la plus belle. p. 1 i2.

La purpre de Tayente a éli la plus estimés. pendant quelque tems. p. x 33.

Auteurs anciens qui ont parlé de la pourpre. p. 114.

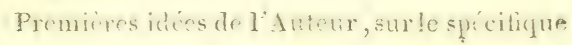
anti-herniaire. p. 114.

Le limaçon qui prod̉uisait l'ancienne pourpre, a été retrouvé en Amérique. p. 115.

Procédés dont les modernes font usage pour avoir la couleur pourpre. p. 115. 
L'ancienne pourpre a été aussi retrourcée cir Angleterre et en France. p. 117.

\section{A R T I CLE II.}

RECHERCIIES SUR LE SUC DES ESCARGOTS DE VIGNE. p. 119 .

Essais de teinture, arec le suc d'escargots de vigne. p. 119 .

Conjectures médicinales, sur le suc de ces escargots. p. 119.

Découverte du spécique anti-hemiaire, incomnu jusqu'ì ce jour. p. 120.

\section{A R T I CLE III.}

ESSAIS SUTR LA GUÉRTSON DES IIERNTES. 1). 121 .

Gućrison de deux hernies, en la personne d'un jeune homme de 24 ans, et d'un autre de 36 ans. p. 121.

Propositions faites au plus célèbre chirurgien herniatere de Paris, relativement ì la découverte du spécifique, p. 121.

Réponse blamable de ce chirurgien. p. 122. 


\section{DES MA TIERES. 167}

Trois nouvelles gurisons de hernics. p. 122.

Hommage fait à l'humanité du secret de 'Auteur. p. 124.

\section{A R T I L E IV.}

TRATTERENT DES HERNIES. P. 125.

Consultation preliminire que doil prendre be malade, auprès d'un homme de l'art. p. 125.

Idée générale de celle consultation. p. 125 .

Cas où le spécifique ne peut êlre d'aucun usage. p. 125.

Description d'un bandage à coquille, pour la gnúrison des hernies, en faisant usage du spécifique. p. 126.

Descriplion d'un godet propre à recevoir lo spécifique, p. 127.

Maticres propres à maintenir le spécifique daus le godel. p. 128 .

La laine est préférable au coton et au lin. p. 128 .

A perçu du nombre d'escargots de vigne nicessaire da la guérison radicale d'une hernice, p. 129 .

Si lon employail d'aulres escirgots, ils s! 
raient igalement bins, mais il en laudrant beaucoup plus. p. 129 , note 6 .

Lieux où se trouvent les escargots de vigue. p. 129 .

Choix qu'on en doit faire, pour le traitement des hernies. p. 150.

Le printems est la saison la plus arantageuse et la plus favorable à ce traitement. p. 130.

Raisons de celte préférence. p. 150.

Moyen d'ćconomiser les escargols. p. 131.

Comment on extrait le spécifique du corps des escargols. p. 131 .

Le spécifique esi le sang des escargots. p. 152.

Description du coeur des escargols. p. 152.

Le cour des escargots, arraché de leur corps, conserve son mourement péristaltique, pendant plus de cinq minutes. p. 155.

Anaiomie de la limace et du colimaçon, par George-Curier, secrétaire perpétuel de l'Instilut. p. 135.

Terme que l'on doit mettre à l'extrail du spécifique, dans chaque escargot. p. 135.

Nombre d'escargols de rigne nécessaire à chaque pansement. p. $15 \%$ 


\section{DES MATERES. 169}

Calcul exact du nombre d'escargols de rigne nécessaire à la gुnérison radicale d'une hernie, sans emplover le moyen économique proposé ci-dessus. p. 154 .

Comment on domne une consistance solide au spécifique, quand on manque de laine, pour le maintenir dans le godet. p. 155.

Ce procédé ne doit point laire négliger l'usagr de la laine. p. 155.

Une laine trop long-tems gardée sur la hernie, pourrait introdnire, dans le bas-ventre, mne corruption très-dangereuse. p. 155.

Méthode des pansemens. p. 156.

Réflexion sur les taches que le spécifique fait au linge. p. 157 .

Conduite du malade durant le traitement de sa hernic. p. 158.

\section{A RTICI.H V.}

RÉSULTAT. p. 140.

Durée du trailement, on général. p. 140.

Manière de reconuaître l'efficacité du spécilique, et la guérison radicale d'une heruie. p. 140 . 
170

\section{E $\mathrm{BLE}$}

Lanature peal acierer sente cette gnérison, guand elle tonche à sa fin. n. 140.

$$
\text { A RTICLE VI. }
$$

INSTAVCTION SUR LA CONVAIESCENCE. ?. 141 .

Nicessite cic perer un bandage, penciint entinon deux mois, apres la guérisou dine luervie. p. 141 .

Les raisons de cette première ordonnance. j. 141.

Alcution que doit aroir le malade de forlifer l'annean avee sa main, dans tous les efforts fut'il peut faire, apre's aroir quitté son bandage, dans les premiers tems de sa guérison. p. 142 ,

La raison de celle seconde ordonnance. p. 142 .

Quelle que soit la situation morale el physique du conralescent, sa gućrison est assurée, a's qu'il n'est plus dans le cas de faire usage du spécifique. p. 144.

Remarque générale sur les suiles du trailement. p. 144 . 
DES M A T I R ES. 171

L'Auteur prévoit qu'il sera atlaqué par de mauvaises passions. p. 145.

Dernier mot à ce sujet. p. 146, à la fin.

\section{F I N}

De la Table des Matières. 


